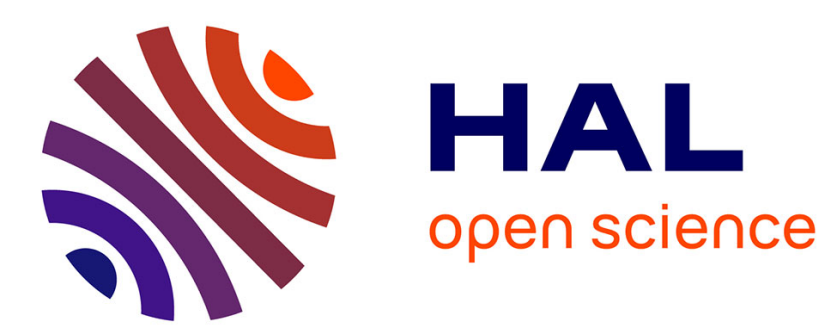

\title{
Numerical study of screech generation in a planar supersonic jet
}

Julien Berland, Christophe Bogey, Christophe Bailly

\section{To cite this version:}

Julien Berland, Christophe Bogey, Christophe Bailly. Numerical study of screech generation in a planar supersonic jet. Physics of Fluids, 2007, 19 (7), pp.075105. 10.1063/1.2747225 . hal-02342186

\section{HAL Id: hal-02342186 \\ https://hal.science/hal-02342186}

Submitted on 31 Oct 2019

HAL is a multi-disciplinary open access archive for the deposit and dissemination of scientific research documents, whether they are published or not. The documents may come from teaching and research institutions in France or abroad, or from public or private research centers.
L'archive ouverte pluridisciplinaire HAL, est destinée au dépôt et à la diffusion de documents scientifiques de niveau recherche, publiés ou non, émanant des établissements d'enseignement et de recherche français ou étrangers, des laboratoires publics ou privés. 


\title{
Numerical study of screech generation in a planar supersonic jet
}

\author{
Julien Berland, ${ }^{\text {a) }}$ Christophe Bogey, and Christophe Bailly \\ Laboratoire de Mécanique des Fluides et d'Acoustique, UMR CNRS 5509, \\ Ecole Centrale de Lyon, 69134 Ecully, France
}

(Received 2 August 2006; accepted 19 April 2007; published online 19 July 2007)

\begin{abstract}
The generation of screech tones in an underexpanded jet is investigated by means of compressible large eddy simulation (LES). A three-dimensional planar geometry is considered with the aim of studying screech radiation in a simple jet configuration, whose physics nevertheless remains similar to that of large-aspect-ratio rectangular jets encountered in experimental surveys. The jet operates at fully expanded Mach number $\mathrm{M}_{j}=1.55$, with Reynolds number $\mathrm{Re}_{h}=6 \times 10^{4}$. The LES strategy is based on explicit selective filtering with spectral-like resolution, and low-dispersion and low-dissipation numerical algorithms are implemented to allow the direct noise computation of the phenomenon. The numerical results are first set against experimental data to establish the consistency of the simulation. It is shown that the flow development and the shock-cell structure are in agreement with experiments of the literature. Furthermore, the upstream acoustic field exhibit harmonic tones that compare correctly to screech tones observed on rectangular jets in terms of frequency, amplitude, and phase shift on either side of the jet. The extrapolation of the LES near-field data to the far field demonstrates that the radiated noise includes the three characteristic noise sources of supersonic shock-containing jets: screech tones, shock-associated noise, and mixing noise, with frequencies and directivities in agreement with experimental observations. Flow visualization of shock/vortex interactions near the third shock-cell finally provides evidences that screech sound sources can be interpreted using the shock-leakage theory. It is shown in particular that the third compression shock within the jet can leak outside through regions of the shear layer with low level of vorticity and results in the production of upstream-propagating sound waves contributing to screech tone radiation. (c) 2007 American Institute of Physics.
\end{abstract}

[DOI: $10.1063 / 1.2747225]$

\section{INTRODUCTION}

Under certain operating conditions, supersonic over- and underexpanded jets can produce discrete frequency sounds, referred to as screech tones, dominating all other noise sources in the forward direction. This phenomenon was first observed in the 1950s by Powell, ${ }^{1}$ who proposed that the screech production is controlled by a feedback loop. The interactions of the turbulent motions developing in the jet shear layers with the shock waves of the quasi-periodic shock-cell system may indeed give rise to upstreampropagating acoustic waves. The resonant loop is closed at the nozzle lip where sound waves are reflected back and excite the shear layer.

Since the first observations of Powell a large number of experimental and theoretical studies have been carried out and knowledge has been gained on the self-sustained loop characteristics. One may refer for instance to the review of $\operatorname{Raman}^{2}$ for further details. Current understanding of the screech is nevertheless primarily qualitative, and quantitative predictions are limited to the fundamental frequency at which the phenomenon occurs. There is, in particular, no theory that permits to determine the amplitude of the upstream-propagating acoustic fluctuations. Such a predic-

\footnotetext{
${ }^{a)}$ Author to whom correspondence should be addressed. Mailing address: Centre Acoustique, Ecole Centrale de Lyon, 36 avenue Guy de Collongue, 69134 Ecully, France. Electronic mail: julien.berland@ec-lyon.fr
}

tion relies on a clear understanding of the sound generation by shock/vortex interactions inside the shear layer. Recent work by Suzuki and Lele ${ }^{3}$ introduced geometrical acoustics to model the sound generation process. An analogy between ray trajectory inside the jet and shock deformation may indeed be drawn to show that shocks tend to leak through the shear layer near the saddle points of vortices, and that this process, moreover, results in the production of sound waves with shapes similar to those of the screech tones. The study of Suzuki and Lele ${ }^{3}$ has, however, been accomplished for a single shock interacting with a shear layer and experimental evidences of the shock-leakage phenomenon are not available so far. Numerical simulations can nonetheless help to demonstrate that shock leakage occurs in screeching jet.

Simulations of jet screech noise have indeed become recently feasible. Such computations remain, nevertheless, a challenging issue: the self-sustained aeroacoustic loop responsible for screech tones leads to a strong coupling between the aerodynamic and the acoustic fluctuations, and both fields therefore have to be resolved within a same run. This method, referred to as direct noise computation (DNC), has in addition difficulty dealing with shock-associated noise simulations because it requires discretization algorithms with high-order properties. Such simulations have to compute shock waves while being able to resolve the full range of length and amplitude scales associated with aerodynamic and acoustic fluctuations, and nonreflecting boundary conditions 
for both turbulent motions and sound waves have to be implemented.

Shen and $\mathrm{Tam}^{4}$ and $\mathrm{Li}$ and $\mathrm{GaO}^{5}$ solved, for example, the unsteady Reynolds-averaged Navier-Stokes equations to reproduce the screech tones of circular jets. Their method, however, provides a phase-averaged solution at the forcing frequency induced by the screech, and thus simulates a limited bandwidth of the turbulence spectra. The full range of turbulence scales can be obtained by direct numerical simulations (DNS). The required computational effort is, unfortunately, very important and flow investigation using DNS is generally restricted to low Reynolds number phenomena. In an attempt to study shock-associated noise, Suzuki and Lele ${ }^{3}$ have, for instance, performed DNS of a supersonic shear layer impinging on an oblique compression wave with the aim of providing an insightful investigation of the shockleakage phenomena. However, such a DNS can hardly be extended to a complete shock-containing supersonic jet. The study of flows with Reynolds number of practical interest, and of their radiated acoustic fields may be performed using compressible large eddy simulation (LES). In LES, only the larger scales are resolved and a subgrid scale model takes into account the effects of unresolved small wavelengths. It is therefore possible to deal with realistic turbulence configurations while keeping computational cost at a reasonable level. Feasibility of DNC of subsonic jets using LES has been demonstrated, for instance, by Bogey and Bailly ${ }^{6,7}$ and by Bodony and Lele. ${ }^{8}$ As for LES of supersonic jet noise, Al Qadi and Scott $^{9}$ performed LES of a rectangular jet exhibiting screech tones, the shocks being captured using a highorder filter combined to a total variation diminishing (TVD) time integration scheme. Imamoglu and Balakumar ${ }^{10}$ carried out the computation of a shock-containing circular jet using implicit LES based on weighted essentially nonoscillatory spatial discretization techniques, and on a TVD algorithm for time advancement. Loh et al. ${ }^{11}$ also simulated the screech tones of a circular jet using a low-order finite volume method. The simulations above, however, involve shock capturing or low-order algorithms, and these methods have been shown not to be especially appropriate for LES because they may introduce excessive dissipation on the resolved scales, ${ }^{12}$ or may generate numerical errors with large magnitude. ${ }^{13}$ The alternative is thus to perform a LES relying only on low-dispersion and low-dissipation algorithms, which ensure numerical accuracy while avoiding significant artificial damping of the wavelengths accurately resolved. ${ }^{14}$

In the present study, the compressible LES of a planar shock-containing jet is performed with spectral-like algorithms. The jet operates at underexpanded conditions so that a quasi-periodic shock-cell structure is formed in the jet plume. The Reynolds number based on the fully expanded jet Mach number $\mathrm{M}_{j}=1.55$, and on the jet height $h=3 \mathrm{~mm}$, is equal to $6 \times 10^{4}$. The LES approach is based on lowdispersion and low-dissipation numerical methods, ${ }^{15,16}$ and selective filtering is applied to take into account the effects of the subgrid scales. ${ }^{14}$ The objective is to provide evidence using DNC that the shock-leakage phenomenon may be responsible for screech sound generation in a supersonic jet. A three-dimensional planar geometry has been chosen in order to study screech tone radiation in a simple jet configuration which may nevertheless be compared to experimental data, owing to the similarities between the physics of plane jets and the behavior of large aspect ratio rectangular jets. Hence, comparison of LES results with experimental data are first made to show that even though the LES relies on a modelized geometry, the physics of the key elements of the screech feedback loop, namely, the shock-cell structure, the shear layer oscillation mode, and the upstream acoustic field, is correctly reproduced. Once consistency of the simulation is demonstrated, numerical results are used in a prospective manner. The full aeroacoustic field is thus investigated to provide numerical evidences of the shock-leakage phenomenon as being a mechanism of screech noise generation.

The outline of the paper is the following. The numerical procedure and the simulation parameters are detailed in Sec. II. The turbulent flow development and the acoustic field of the LES are then investigated in Sec. III. Flow visualizations of shock/vortex interactions in the shear layer are finally depicted in Sec. IV to provide evidences of the connection between the shock-leakage process and the sound sources of the screech tones. Concluding remarks are drawn in Sec. V.

\section{NUMERICAL PROCEDURE}

\section{A. LES strategy and numerical methods}

The compressible Navier-Stokes equations written in the form proposed by Vreman et al. ${ }^{17}$ are solved to perform the LES of a three-dimensional planar underexpanded jet. To take account of the dissipation provided by the unresolved scales, an eddy-viscosity-based model is commonly introduced. This modeling approach may nevertheless introduce excessive dissipation on the resolved scales, leading to a decrease of the effective Reynolds number of the simulation. ${ }^{18,19}$ An alternative to eddy-viscosity models consists in minimizing the dissipation on the resolved scales. Explicit spectral-like filtering of the flow variables is therefore applied to diffuse energy only at the smaller resolved scales, close the grid cutoff wave number. The method has been successfully used in recent applications ${ }^{6,7,14,20}$ and is used here for a planar supersonic shock-containing jet.

Low-dispersion and low-dissipation explicit numerical schemes designed on 11 points ${ }^{15}$ are used for spatial discretization. The finite differences are of order 4, and the filtering is performed by a second-order scheme. Both discretization algorithms have furthermore been optimized in the Fourier space so that they can propagate accurately perturbations with at least four points per wavelength. The filter exhibits, in particular, a sharp cutoff so that few artificial dissipation is applied to resolved scales. Indeed, by decreasing the formal order of a large stencil scheme, one may freely adjust some of the coefficients of the filtering algorithm to optimize its dissipation properties. Time integration is carried out by an optimized fourth-order low-storage Runge-Kutta scheme, ${ }^{16}$ able to resolve waves with at least four time steps per period. Periodic boundary conditions are implemented in the $z$ direction while nonreflecting conditions of Tam and Dong ${ }^{21}$ are enforced in the $x$ and $y$ directions so that acoustic perturbations can leave the computational domain without the gen- 


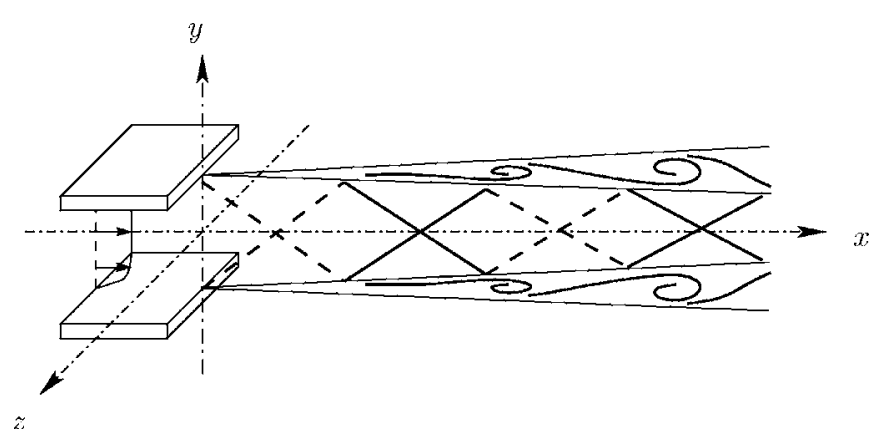

FIG. 1. Sketch of the computational domain and of the coordinate system (the figure is not to scale).

eration of significant spurious waves. In addition, a sponge zone is set up at the outflow in order to attenuate aerodynamic fluctuations before they reach the downstream limit of the domain. The jet nozzle, which is known to be necessary for screech tone emergence, is described by two parallel adiabatic plates separated by a distance $h$ defining the jet height. Close to the solid surfaces noncentered fourth-order 11-point finite differences ${ }^{22}$ are used, and filtering is performed with centered filters for stability purposes, stencil sizes being progressively reduced close to the walls. Pressure and density at the wall are computed with the Navier-Stokes equations in which quantities containing zero velocities are removed. Inside the nozzle, characteristics ${ }^{23}$ are implemented to specify the inflow conditions. A sketch of the computational domain and of the coordinate system is given in Fig. 1.

\section{B. Simulation parameters}

\section{Inflow conditions}

The jet is assumed to be supplied by a convergent nozzle whose designed Mach number $\mathrm{M}_{d}$ is therefore equal to 1 . The ambient region surrounding the flow is at rest and characterized by a pressure $p_{\infty}=10^{5} \mathrm{~Pa}$ and a density $\rho_{\infty}$ $=1.22 \mathrm{~kg} \mathrm{~m}^{-3}$. Reservoir temperature $T_{r}$ is set to $288 \mathrm{~K}$, and an elevated pressure $p_{e} / p_{\infty}=2.09$ is imposed inside the nozzle so that the jet operates at underexpanded conditions. The fully expanded jet Mach number $\mathbf{M}_{j}$, defined by

$$
\mathbf{M}_{j}=\left\{\frac{2}{\gamma-1}\left[\left(1+\frac{\gamma-1}{2} \mathrm{M}_{d}^{2}\right)\left(\frac{p_{e}}{p_{\infty}}\right)^{(\gamma-1) / \gamma}-1\right]\right\}^{1 / 2}
$$

is then equal to 1.55 . This value has indeed been observed by Krothapalli et al. $^{24}$ to correspond to maximum screech sound radiation for a rectangular jet with a large aspect ratio.

Inside the nozzle, flow variables are uniform except near the walls, where a no-slip condition is required. Boundary layer profiles are therefore implemented close to the solid surfaces using an approximation of the laminar Blasius solution, given by

$$
\frac{u}{U_{e}}= \begin{cases}\eta\left(2-2 \eta^{2}+\eta^{3}\right) & \text { if } \eta<1, \\ 1 & \text { if } \eta \geq 1,\end{cases}
$$

where $\eta$ is the distance to the wall normalized by the boundary layer thickness $\delta$, and $U_{e}=310 \mathrm{~m} \mathrm{~s}^{-1}$ is the designed nozzle velocity. The inflow density profile is then given by the Crocco-Busemann relation:

$$
\rho=\left[\frac{1}{\rho_{w}}-\left(\frac{1}{\rho_{w}}-\frac{1}{\rho_{e}}\right) \frac{u}{U_{e}}-\frac{\gamma-1}{2} \mathrm{M}_{d}^{2}\left(\frac{u}{U_{e}}-1\right) \frac{u}{U_{e}}\right]^{-1},
$$

where the density on the walls $\rho_{w}$ can be evaluated by the perfect gas relationship $\rho_{w}=p_{e} / r T_{r}$. For high Reynolds number around $10^{5}$, boundary layers are generally very thin ${ }^{25}$ with a momentum thickness $\delta_{\theta} / h$ of the order of $10^{-3}$. However, to perform a computation with an affordable number of grid points, $\delta_{\theta} / h$ is set in this work to a larger value: 3 $\times 10^{-2}$. Discrepancies between the LES results and the experimental data of the literature are consequently expected, and the momentum thickness has to be kept in mind while interpreting numerical results.

The jet height is $h=3 \mathrm{~mm}$ and the nozzle lip thickness $h_{l}$ is similar to that used in the experiments of Raman and Rice, ${ }^{26}$ that is, $h_{l}=h / 4$. The Reynolds number $\operatorname{Re}_{h}=U_{j} h / \nu$ is then $6 \times 10^{4}$, where $U_{j}=\mathrm{M}_{j} c_{j}$ with $c_{j}=278 \mathrm{~m} \mathrm{~s}^{-1}$.

\section{Numerical parameters}

The computational domain is discretized by a Cartesian grid of $525 \times 257 \times 121=16.3 \times 10^{6}$ points. The smallest mesh size $\Delta_{m}=h / 40$ allows us to use 40 mesh points within the jet height and seven nodes inside the boundary layer. The domain of interest, which excludes the sponge zone, has dimensions $25.6 h \times 16 h \times 5 h$, with a nozzle extending over $0.6 h$ inside the domain. The time step $\Delta t \simeq 8.6 \times 10^{-8}$ corresponds to a $\mathrm{CFL}=\left(U_{j}+c_{j}\right) \Delta t / \Delta_{m}$ number equal to 1 (where CFL denotes the Courant-Friedricks-Levy number) and selective filtering is applied every iteration. To ensure statistical convergence and to compute at least 100 periods of the screech loop, the simulation is run over 80000 time steps.

\section{RESULTS AND VALIDATION}

\section{A. Instantaneous vorticity and pressure}

An instantaneous snapshot of isosurfaces of the spanwise vorticity $\omega_{z}$ in the whole computational domain is represented in Fig. 2. A large range of turbulence scales, especially the fine scales characterizing high Reynolds number flows, are observed. Pressure isocontours in the plane $z / h$ $=-2.5$ are also reported. Upstream-propagating wavefronts are clearly visible on both sides of the jet. This acoustic radiation is typical of screech tones, and it will be shown in Sec. III D that the phenomenon is locked on a frequency denoted $f_{s}$, whose Strouhal number $\mathrm{St}=f_{s} h / U_{j}$ is equal to 0.126 .

\section{B. Mean flow field}

\section{Global mean flow}

A colormap of the normalized mean streamwise velocity $\bar{u} / U_{j}$ in the central $(x, y)$ plane is shown in Fig. 3. Due to the overpressure at the nozzle exit, five shock-cells exhibiting the distinctive bow-shaped structure are apparent in the jet plume for $x / h<10$. In addition, a decrease of the axial velocity and a weakening of the shock strength in the downstream direction are observed. The interactions between the 


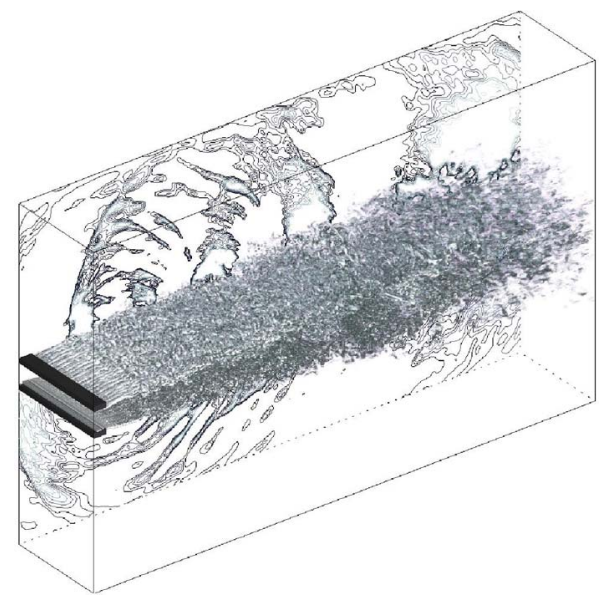

FIG. 2. Instantaneous snapshot of spanwise vorticity $\omega_{z}$ in the whole computational domain and of pressure in the plane $z / h=-2.5$. The nozzle lips are represented in gray.

cells and the shear layer are indeed stronger as the vortical structures develop in the streamwise direction. The velocity and pressure gradients induced by the shocks are consequently smoothed out. Also shown in Fig. 3 is the supersonic core of the jet, whose boundary is defined by the sonic line where the Mach number $\mathrm{M}$ is 1 . This region extends down to $x / h=22$, which is somewhat larger than the value of $x / h$ $=15$ measured by Krothapalli et al. ${ }^{24}$ for a large-aspect-ratio rectangular jet operating at $\mathrm{M}_{j}=1.52$, with a Reynolds number based on the nozzle exit velocity of about $10^{5}$. This discrepancy may be attributed to the turbulent development of the jet. The initial shear layer of the jet simulated by LES is indeed laminar and relatively large. The shear layer at the nozzle exit of the experiments of Krothapalli et al. ${ }^{24}$ is, however, probably thinner and turbulent, which may lead to a faster jet development and to a stronger decay of the mean centerline velocity with respect to the simulation.

\section{Shock-cell structure}

The shock-cell spacing $L_{s} / h$ is presented in Fig. 4 as a function of the fully expanded jet Mach number $\mathrm{M}_{j}$ for various experiments on rectangular jets. Also plotted is the average spacing between the five shock-cells visible on the LES mean flow data. The shock-cell spacing provided by the computation appears to be slightly smaller than expected. This trend may be explained by the initial shear layer thick-

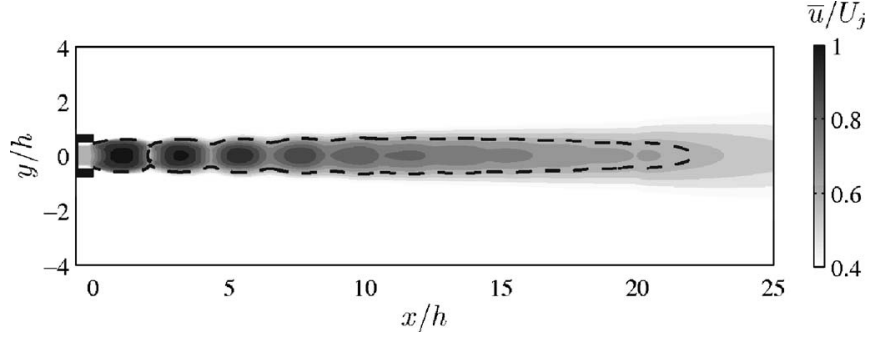

FIG. 3. Colormap of the normalized mean streamwise velocity $\bar{u} / U_{j}$ in the central $(x, y)$ plane. - - -, sonic line $(\mathrm{M}=1)$. The black rectangle represents the nozzle lips.

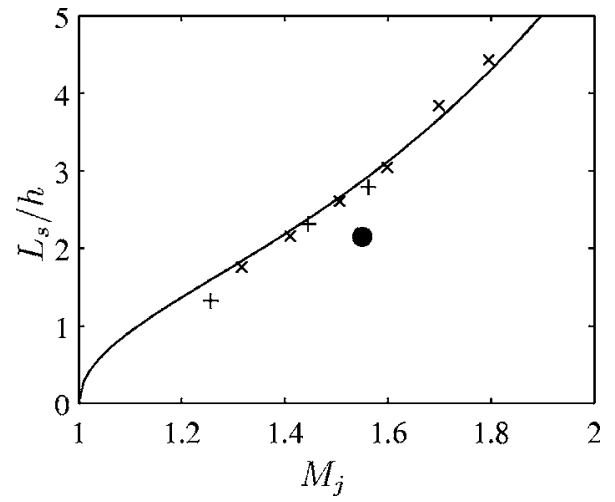

FIG. 4. Shock-cell spacing $L_{s} / h$ as a function of the fully expanded jet Mach number $\mathrm{M}_{j}$. Measurements on rectangular jets: +, Raman and Rice (Ref. 26); ×, Panda et al. (Ref. 27); — , analytical solution of Tam (Ref. 28) for a plane jet; $\bullet$, present computation.

ness used for the calculation. Indeed, according to Tam, ${ }^{28}$ the jet column acts as a waveguide for flow discontinuities, and the successive reflections of shocks and expansion waves on the sonic line give rise to the periodic shock-cell structure. Shock spacing is therefore related to the height of the supersonic core, which is relatively small in the present jet due to the thickness of the shear layer. The supersonic core height at the nozzle exit is, more precisely, $h^{*} \simeq 0.8 h$. The normalized shock-cell-spacing $L_{s} / h^{*}$ is then equal to 2.7 , which is in good agreement with the experimental results shown in Fig. 4. This trend is in addition supported by the shock-cell model of Morris and Baht, ${ }^{29}$ which shows in particular that shock spacing of round jets decreases when the mixing layer thickness is increased.

The strength $\left(p_{1} / p_{\infty}-1\right)$, where $p_{1}$ is the mean pressure just upstream of the shock, of the first three shocks of the simulation are compared in Fig. 5 with the measurements of $\operatorname{Raman}^{30}$ obtained for a supersonic rectangular jet at the same Mach number. The strength of the first shock is found to agree with the result of Raman, ${ }^{30}$ whereas the second and the third shocks exhibit a larger pressure gap. The overestimation of the shock strengths may have two origins. First, as it has been previously reported in this section, the present nozzle inflow conditions probably induce a slower turbulent flow development, implying a slower weakening of the

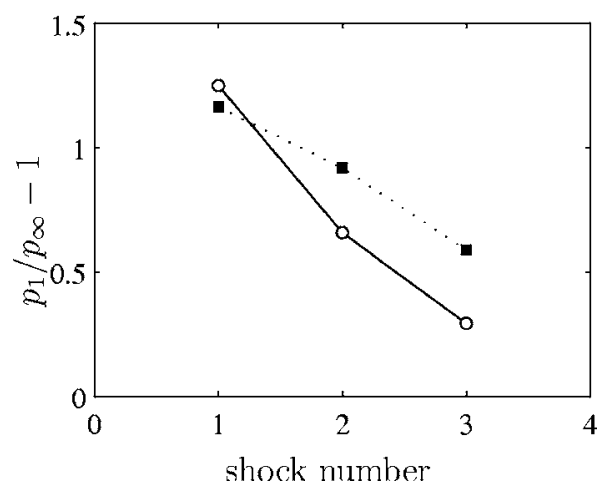

FIG. 5. Shock strength $p_{1} / p_{\infty}-1$ for the first three shock-cells ( $p_{1}$ is the pressure upstream the shock). $\mathbf{\square}$, Raman (Ref. 30) measurements on a $\mathbf{M}_{j}$ $=1.55$ rectangular jet; $\cdots \mathbf{\square} \cdots$, present computation. 


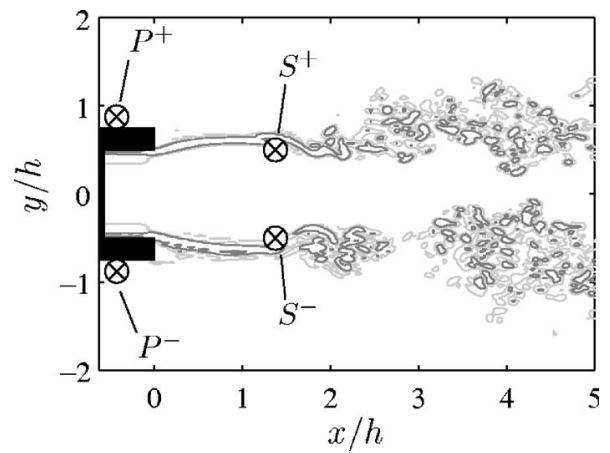

FIG. 6. Locations of the measurement points in the central $(x, y)$ plane. $S^{+}$ and $S^{-}$: velocity measurements for the investigation of the shear layer oscillation mode; $P^{+}$and $P^{-}$: pressure measurements to study the upstream acoustic field.

shocks compared to the experiment of Raman. ${ }^{30}$ One may also argue that the shear layer development in the major-axis plane of rectangular jets contributes to three dimensional shock-cell structures ${ }^{31}$ whereas for planar jet the mean flow is homogeneous in the spanwise direction. This observation could support the fact that the first shock is similar in rectangular and planar jets. However, further downstream, the three-dimensional structure of the jets should differ, resulting in discrepancies between shock strengths.

\section{Shear layer oscillation modes}

The shear layer development is now investigated using velocity fluctuations provided by the LES, with the aim of characterizing the development of the aerodynamic instabilities.

\section{Dominant instability modes}

The axial evolution of the instability modes is first studied. Spectra of axial and transverse velocity perturbations $u^{\prime}$ and $v^{\prime}$ are computed in the shear layer along the line $(x, y$ $=h / 2, z=0$ ), as shown in Fig. 6, where the locations of the measurement points are reported. The streamwise development of the power spectral density of the velocity component $u^{\prime}$ is represented by the colormap of Fig. 7(a) as a function of the axial position $x / h$. In the upstream part of the flow, for $x / h<10$, the dominant mode is observed to be locked on the screech Strouhal number $\mathrm{St}=0.126$. In addition, the amplitude of velocity fluctuations for this mode exhibits a significant modulation by the shock-cell structure, which is indicated by the successive decrease and recovery of the power spectral level close the shocks. Further downstream, for $x / h>15$, the instability wave at the screech frequency vanishes, and a lower-frequency mode with a broader spectral distribution is seen to develop in the shear layer. The peak frequency is, moreover, shown to slightly decrease with $x / h$. Indeed, the corresponding Strouhal number is, for instance, equal to 0.054 in $x / h=15$, and to 0.036 in $x / h=25$. Note that these frequencies are not subharmonic of the screech tones.

The axial evolution of the power spectral density of $v^{\prime}$ is now presented in Fig. 7(b). Similar to the velocity component $u^{\prime}$, the dominant oscillation mode of the shear layer occurs at the fundamental screech frequency. The instability
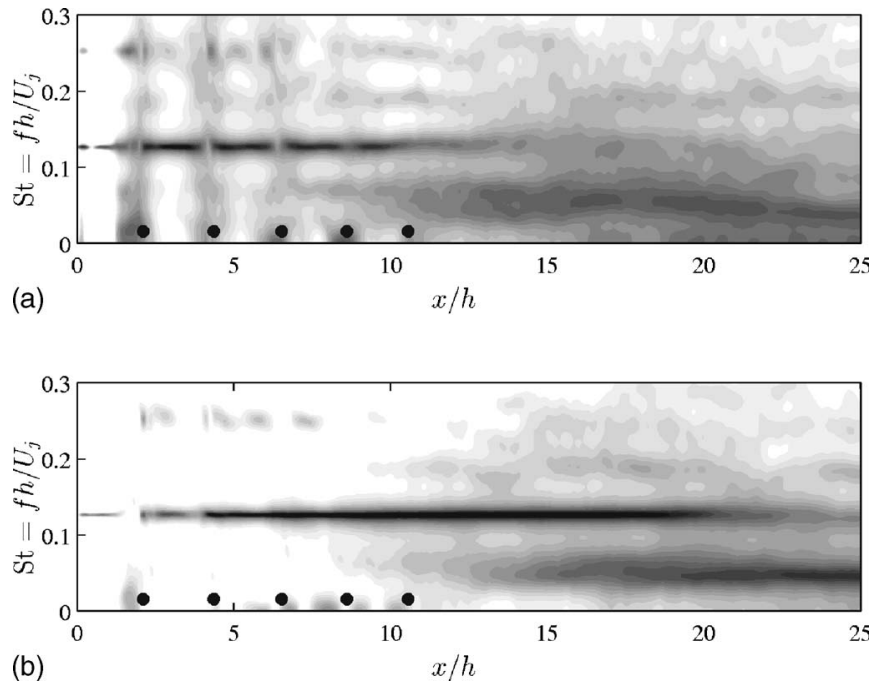

FIG. 7. Streamwise development of the power spectral density of the velocity fluctuations in the shear layer, along the line $(x, y=h / 2, z=0)$, in logarithmic scales. The spectra are normalized using the jet velocity $U_{j}$. (a) Axial perturbations $u^{\prime}$ and (b) transverse fluctuations $v^{\prime}$. The shock locations deduced from the mean flow are represented by the symbols

mode nevertheless shows a weaker modulation by the shocks and is in addition clearly observed up to $x / h=22$. For $x / h$ $>15$, a low-frequency mode similar to the one observed for $u^{\prime}$ emerges, but with a fairly constant Strouhal number, equal to 0.045 at $x / h=25$.

\section{Transverse symmetry of the modes}

Screech instability modes identified in the upstream part of the jet may be classified using the interpretation reported by Thomas and Prakash ${ }^{32}$ of the sinuous and varicose instabilities of the planar jet. Cross-spectra of the velocity fluctuations on either the jet at $x / h=1.3$, in the shear layer, are therefore determined using the data at the locations $(x / h, y / h)=(1.3,0.5)$ and $(x / h, y / h)=(1.3,-0.5)$, as shown in Fig. 6 by the points $S^{+}$and $S^{-}$, respectively. The crossspectrum based on the axial perturbations $u^{\prime}$ is denoted $P_{u u}$, while $P_{v v}$ is the cross-spectra determined from $v^{\prime}$. The quantities deduced from the cross-spectrum are the amplitudes $|P|$, the coherence functions $C$, and the phases $\varphi$. The amplitude and the phase are respectively provided in Figs. 8(a) and 8(b) for the axial velocity fluctuations $u^{\prime}$, and in Figs. 8(c) and $8(\mathrm{~d})$ for the transverse perturbations $v^{\prime}$. Values taken at the screech harmonic frequencies by the coherence functions $C_{u u}$ based on $u^{\prime}$ and $C_{v v}$, computed from $v^{\prime}$, are given in Table I.

The amplitude $\left|P_{u u}\right|$ shows four harmonic peaks whose fundamental corresponds to the screech frequency. In addition, the coherence function $C_{u u}$ appears to range between 0.9 and 1 for these four frequencies, demonstrating that velocity fluctuations at the screech harmonics are coherent on either side of the jet. The phase $\varphi_{u u}$, which indicates the phase difference between fluctuations in $y=h / 2$ and $y$ $=-h / 2$, shows that the fundamental $\left(f_{s}\right)$ and the second $\left(3 f_{s}\right)$ harmonics are antisymmetric, whereas the first $\left(2 f_{s}\right)$ and the third $\left(4 f_{s}\right)$ harmonics are symmetric. It is indeed observed that $\varphi_{u u}\left(f_{s}\right)=-\pi, \varphi_{u u}\left(2 f_{s}\right)=0, \varphi_{u u}\left(3 f_{s}\right)=\pi$, and $\varphi_{u u}\left(4 f_{s}\right)=0$. 

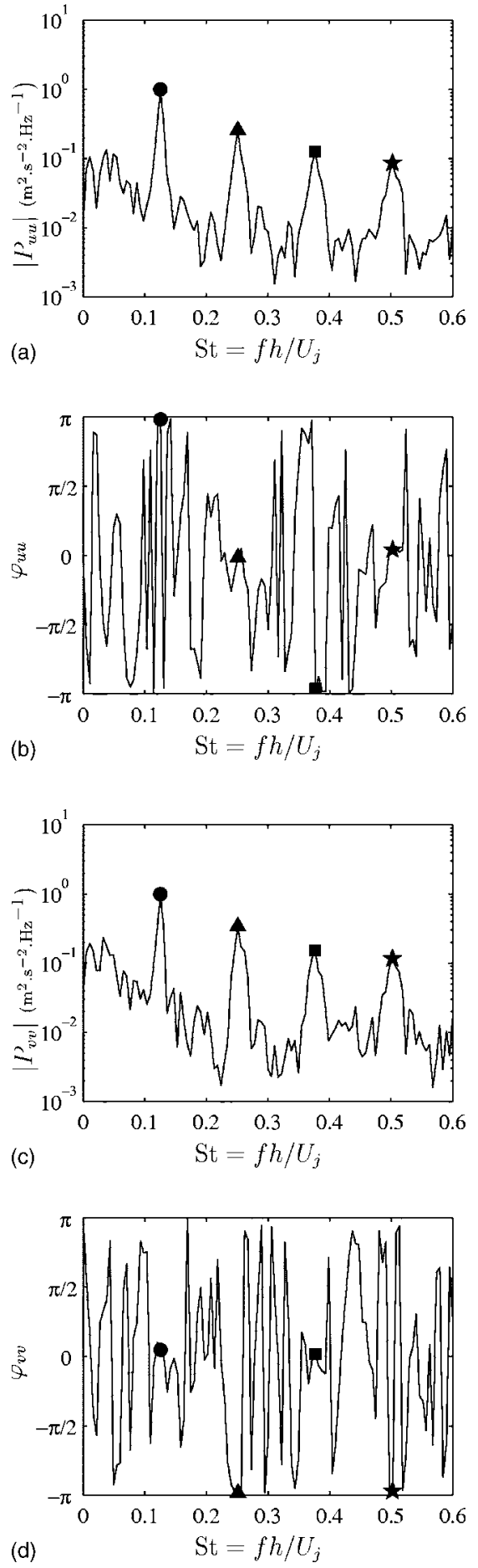

FIG. 8. Cross-spectra of the velocity fluctuations on either side of the jet, in $(x / h, y / h)=(1.3,0.5)$, and $(x / h, y / h)=(1.3,-0.5)$, as functions of the Strouhal number $\mathrm{St}=f h / U_{j}$. $\bullet$, screech frequency $\left(f_{s}\right) ; \boldsymbol{\Lambda}$, first harmonic $\left(2 f_{s}\right)$; $\boldsymbol{\square}$, second harmonic $\left(3 f_{s}\right) ; \star$, third harmonic $\left(4 f_{s}\right)$. Cross-spectrum of the axial velocity perturbations $u^{\prime}$ : (a) amplitude $|P|$; (b) phase $\varphi$. Crossspectrum of the axial velocity perturbations $v^{\prime}$ : (c), amplitude $|P|$; (d), phase $\varphi$.

Consider now the cross-spectrum calculated from the transverse fluctuations $v^{\prime}$, it is found that the amplitude $\left|P_{v v}\right|$ is similar to that obtained for $u^{\prime}$. A series of harmonic peaks are visible in Fig. 8(c), and are coherent since the coherence function $C_{v v}$ displays a large value for these frequencies. The cross-spectrum phase $\varphi_{v v}$ is nevertheless different from $\varphi_{u u}$.
TABLE I. Values taken by the coherence functions $C_{u u}, C_{v v}$, and $C_{p p}$ respectively deduced from the cross-spectrum $P_{u \mu}$ based on the axial velocity perturbations in the shear layer at $S^{+}$and $S^{-}, P_{v v}$ computed with the transverse velocity perturbations in the shear layer at $S^{+}$and $S^{-}$, and $P_{p p}$ based on the pressure perturbations in the upstream direction at $P^{+}$and $P^{-}$(see Fig. 6 for locations of measurement points).

\begin{tabular}{lcccc}
\hline \hline & $f_{s}$ & $2 f_{s}$ & $3 f_{s}$ & $4 f_{s}$ \\
\hline$C_{u u}$ & 0.95 & 0.92 & 0.91 & 0.92 \\
$C_{v v}$ & 0.92 & 0.85 & 0.76 & 0.90 \\
$C_{p p}$ & 1.00 & 1.00 & 0.99 & 0.97 \\
\hline \hline
\end{tabular}

The fundamental $\left(f_{s}\right)$ and the second $\left(3 f_{s}\right)$ harmonics are symmetric, while the first $\left(2 f_{s}\right)$ and the third $\left(4 f_{s}\right)$ are antisymmetric. The phase difference in Fig. $8(\mathrm{~d})$ is such that $\varphi_{v v}\left(f_{s}\right)=0, \varphi_{v v}\left(2 f_{s}\right)=-\pi, \varphi_{u u}\left(3 f_{s}\right)=0$, and $\varphi_{u u}\left(4 f_{s}\right)=\pi$.

It can be finally deduced from the phase differences on either side of the jet that the oscillation modes at the screech fundamental frequency $\left(f_{s}\right)$ and at the second harmonic $\left(3 f_{s}\right)$ are sinuous instabilities, whereas the jet undergoes varicose modes at the first $\left(2 f_{s}\right)$ and third $\left(4 f_{s}\right)$ harmonic frequencies. It should be noted that the oscillation modes at $f_{s}, 2 f_{s}$, and $3 f_{s}$ identified by Raman and Rice ${ }^{26}$ on a screeching rectangular jet have the same phase distribution.

A similar investigation has been carried out for the lowfrequency mode observed in the downstream region of the jet at $x / h=22.5$, for the transverse velocity fluctuations $v^{\prime}$. The cross-spectrum $P_{v v}$ is therefore now computed between the points at $(x / h, y / h)=(22.5,0.5)$ and $(x / h, y / h)=(22.5,-0.5)$.
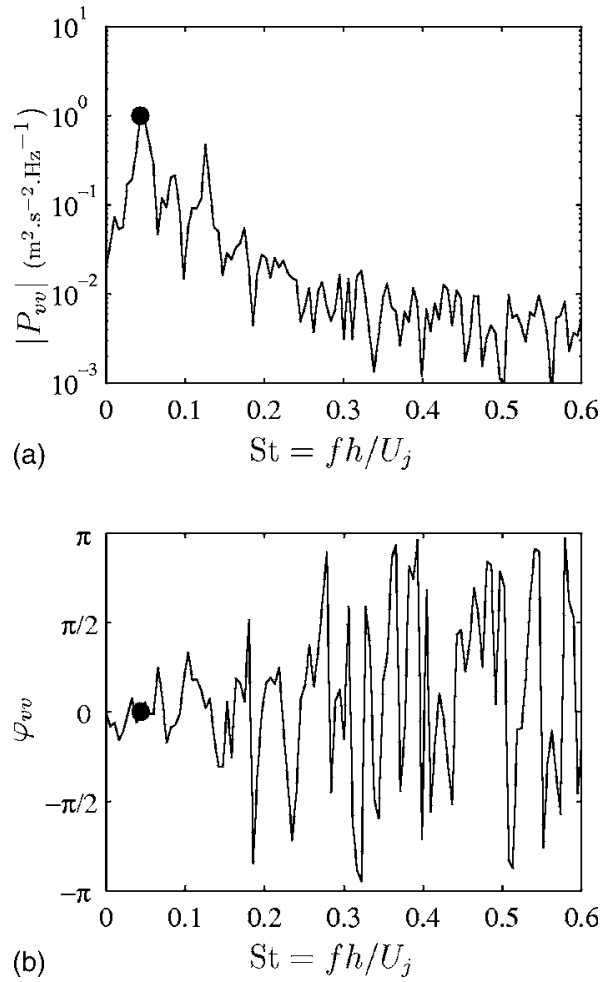

FIG. 9. Cross-spectrum of the velocity fluctuations on either side of the jet, in $(x / h, y / h)=(22.5,0.5)$, and $(x / h, y / h)=(22.5,-0.5)$, as a function of the Strouhal number $\mathrm{St}=f h / U_{j}$., $\mathrm{St}=0.045$. (a) Amplitude $\left|P_{v v}\right| ; \quad$ (b) phase $\varphi_{v v}$. 

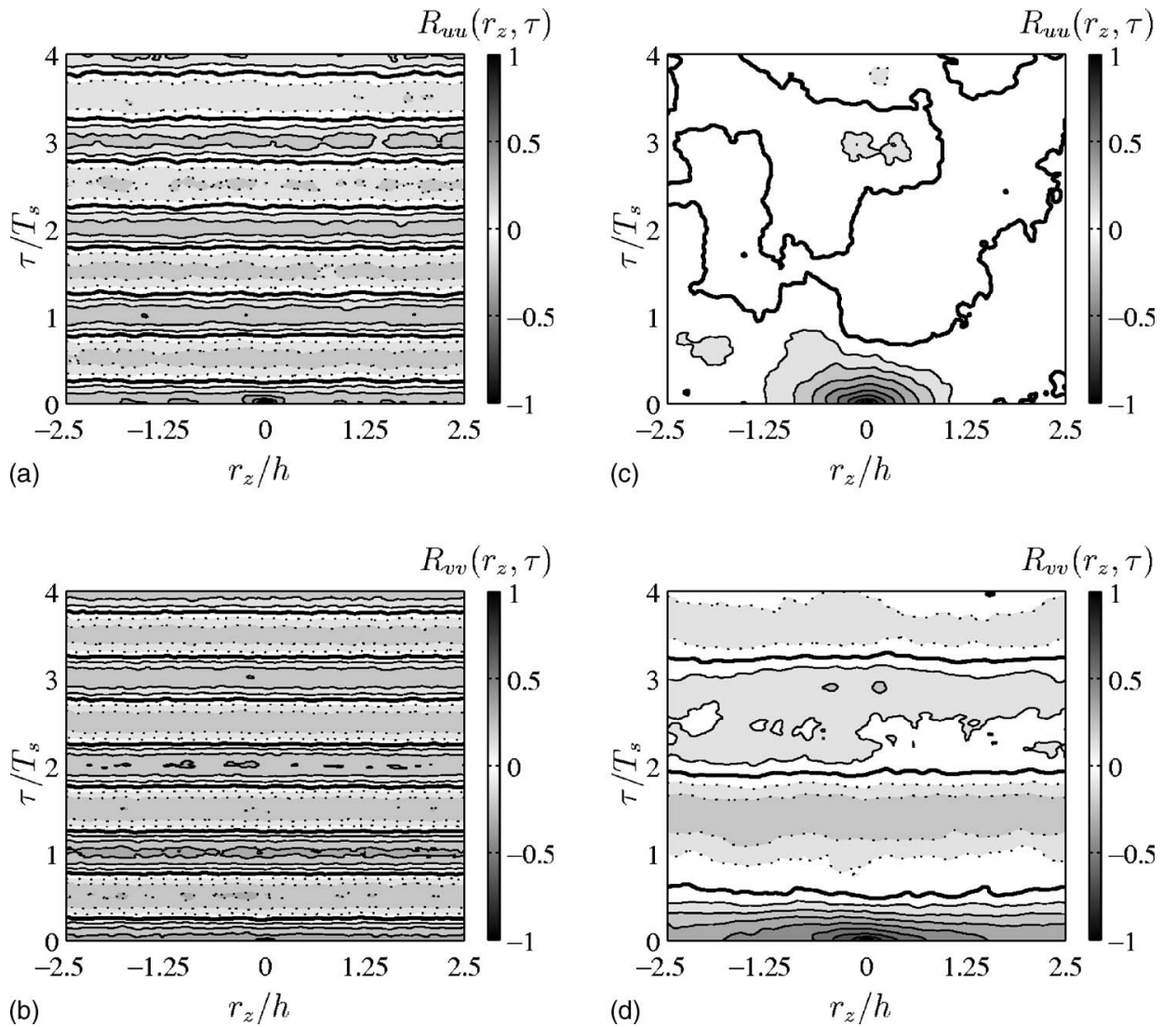

FIG. 10. Space-time correlation coefficients determined in the shear-layer as functions of the spanwise separation $r_{z} / h$ and the time lag $\tau / T_{s}$ normalized by the screech period. Gray scale represents the magnitude of the correlation coefficients and solid and dashed lines denote, respectively, positive and negative values. (a) $R_{u u}\left(r_{z}, \tau\right)$ at $x / h=5$, (b) $R_{v v}\left(r_{z}, \tau\right)$ at $x / h=5,(\mathrm{c})$ $R_{u u}\left(r_{z}, \tau\right)$ at $x / h=22.5$, and (d) $R_{v v}\left(r_{z}, \tau\right)$ at $x / h=22.5$, along the line $(y=0.5 h, z=0) .-, R_{u u}$ or $R_{v v}=0$.
The amplitude $\left|P_{v v}\right|$ and the phase $\varphi_{v v}$ are, respectively, plotted in Figs. 9(a) and 9(b). A peak at Strouhal number St $=f h / U_{j}=0.045$ can be seen in the plot of the amplitude $\left|P_{v v}\right|$. The computation of the coherence function at this frequency yields the value of 0.85 , which shows that the velocity perturbations are correlated on either side of the jet. The phase difference $\varphi_{v v}$ then demonstrates that the low-frequency oscillation mode is symmetric, as $\varphi_{v v}=0$ at $\mathrm{St}=0.045$. The shear layer consequently exhibits a flapping instability mode in the downstream part of the jet.

\section{Spanwise structure}

The spanwise structure inside the shear layer is identified by computing space-time velocity correlations inside the shear layer. The spanwise correlation coefficient based on the axial velocity fluctuations $u^{\prime}$ is defined at $\mathbf{x}$, as

$$
R_{u u}\left(\mathbf{x}, r_{z}, \tau\right)=\frac{\overline{u^{\prime}(\mathbf{x}, t) u^{\prime}\left(\mathbf{x}+r_{z} \mathbf{e}_{z}, t+\tau\right)}}{\left[\overline{u^{\prime 2}(\mathbf{x}, t) u^{\prime 2}\left(\mathbf{x}+r_{z} \mathbf{e}_{z}, t\right)}\right]^{1 / 2}},
$$

where $\mathbf{e}_{z}$ is the unit vector associated with the $z$ direction, $r_{z}$ the spanwise separation, $\tau$ the time lag, and $\cdot$ the statistical average. The coefficient $R_{v v}\left(\mathbf{x}, r_{z}, \tau\right)$ is computed in a manner similar to the transverse perturbations $v^{\prime}$.

The coefficients $R_{u u}$ and $R_{v v}$ are determined at two locations in the shear layer. The first measurement position is located in the upstream part of the jet, at $(x / h, y / h, z / h)$ $=(5,0.5,0)$, in order to investigate the screech oscillation mode. The second point is situated further downstream, at $(x / h, y / h, z / h)=(22.5,0.5,0)$, in order to identify the lowfrequency mode observed in the spectra of Figs. 7(a) and 7(b). Results obtained at $(x / h, y / h, z / h)=(5,0.5,0)$ are plotted in Figs. 10(a) and 10(b) for $R_{u u}$ and $R_{v v}$, respectively, while Figs. 10(c) and 10(d) present the coefficients $R_{u u}$ and $R_{v v}$ at $x / h=22.5$. Magnitude of the correlation coefficients are represented in grayscale, and solid and dashed lines respectively denote positive and negative values. Note that in these figures, the time lag $\tau$ is normalized by the screech period $T_{s}$, and that $r_{z}$ is restricted to the interval $[-2.5 h, 2.5 h]$, which corresponds to the domain extent in the spanwise direction.

Consider first the results at $x / h=5$. As expected, the maximum correlation occurs at $\left(r_{z}, \tau\right)=(0,0)$, where $R_{u u}$ $=R_{v v}=1$. Both correlation coefficients $R_{u u}$ and $R_{v v}$ then quickly decrease as the spanwise separation or the time lag are increased, suggesting the existence of small-scale spatially coherent structures. However, the correlation coefficients do not fall down to zero for large space-time shifts. For instance, for $\tau=0, R_{u u}$ and $R_{v v}$ remain fairly equal to 0.25 when $r_{z} / h$ ranges from -2.5 to 2.5 . In addition, when the time lag $\tau$ is increased, the correlation coefficients show a periodic evolution locked on the period $T_{s}$ of the fundamental screech tone, and do not display significant variations with the spanwise separation $r_{z}$. These results indicate that the oscillation mode of the shear layer at the screech frequency has a two-dimensional structure. This instability is furthermore periodic and strongly correlated in time, which is consistent with the self-excitation due to the screech tones.

The space-time correlations in the downstream region of the jet are now investigated at $x / h=22.5$. The axial velocity correlation $R_{u u}$ in Fig. 10 (c) is equal to 1 at $\left(r_{z}, \tau\right)=(0,0)$. 


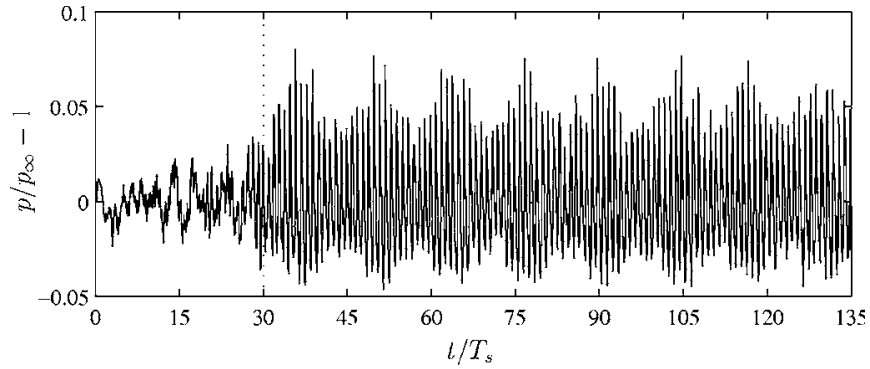

FIG. 11. Pressure history close to the nozzle lip, at $x / h=-0.4$ and $y / h$ $=0.85$. The dotted line represents the end of the transient period of the computation.

When the spanwise separation or the time lag are increased, the magnitude of $R_{u u}$ decreases, but the correlated region is larger than that observed for $x / h=5$. Moreover, for $\left|r_{z}\right|$ $>1.25$ and $\tau>0.5$, the correlation coefficient is close to 0 and the velocity perturbations are therefore weakly correlated. The downstream oscillation mode of the streamwise velocity component $u^{\prime}$ is thus dominated by turbulent motions that do not exhibit any particular spatial or temporal properties. It is not the case for the correlation coefficient $R_{v v}$ of the transverse velocity, which displays in Fig. 10(d) a shape similar to that obtained for $x / h=5$. The coefficient $R_{v v}$ is indeed periodic with the time lag $\tau$, and is fairly constant as the separation $r_{z}$ varies. In addition, the period of the phenomenon corresponds to the low frequency observed in the spectrum of Fig. 7(b), at Strouhal number $\mathrm{St}=0.045$. Note, however, that the correlation in the spanwise direction and in time are weaker than further upstream at $x / h=5$. These results indicate that the downstream region of the jet displays a two-dimensional flapping mode, involving the transverse velocity $v^{\prime}$.

\section{Acoustic field}

\section{Upstream acoustic radiation}

The time evolution of the pressure signal at the upstream location $(x / h, y / h)=(-0.4,0.85)$, represented by the point $P^{+}$ in Fig. 6, is plotted in Fig. 11 as a function of the time $t$ normalized by the screech period $T_{s}$. At the beginning of the computation, for $t / T_{s}<30$, the upstream acoustic field shows a transient behavior. At time $t / T_{s}=30$, the screech feedback is set up, and the pressure signal exhibits periodic oscillations up to the end of the computation, at time $t / T_{s}=135$.

The power spectral density of the pressure perturbations close to the nozzle lip at $P^{+}$is given in Fig. 12 as a function of the Strouhal number $\mathrm{St}=f h / U_{j}$. A series of peaks are visible. The dominant tone is observed at $\mathrm{St}=0.126$ and corresponds to the fundamental screech tone frequency. The other peaks are found to be the harmonics of the phenomenon and have a decreasing energy as the frequency increases. Remark that a nonharmonic peak is also observed in Fig. 12 at the Strouhal number 0.171 . The presence of this discrete frequency, which has not been reported in rectangular jet experiments, is difficult to explain. It is nevertheless interesting to note that this Strouhal number may be interpreted as the sum of the two dominant oscillation modes of the shear layer

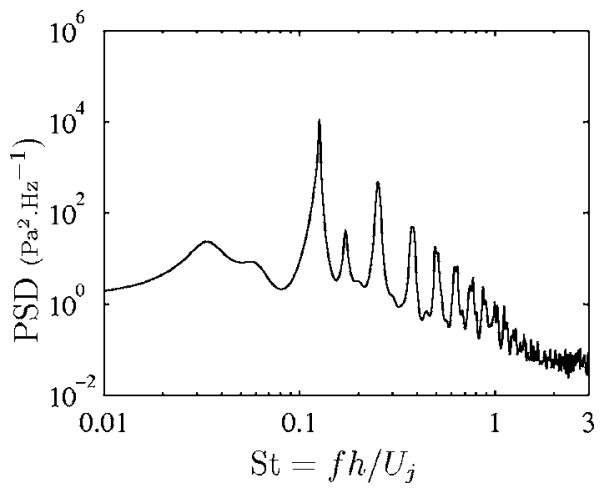

FIG. 12. Power spectral density of the pressure fluctuations close to the nozzle lip, at $(x / h, y / h)=(-0.4,0.85)$, as a function of the Strouhal number $\mathrm{St}=f h / U_{j}$.

reported for $v^{\prime}$ in the downstream direction, shown in Fig. 7(b). Indeed, the low-frequency mode occurs at $\mathrm{St}=0.045$ and the screech mode at $\mathrm{St}=0.126$, leading to a sum equal to 0.171 . Nonlinear interactions between these two modes may thus contribute to a nonharmonic tone radiated in the upstream direction, but the mechanism of sound production has not been identified.

The Strouhal number of the fundamental discrete frequency is now represented in Fig. 13, where it is also plotted against the fully expanded jet Mach number for several experiments on rectangular jets, and where the analytical solution proposed by $\operatorname{Tam}^{28}$ is shown for comparison. The screech Strouhal number provided by the LES is found to be slightly higher than experimental and theoretical results. Indeed, as pointed out in Sec. III B, the shock-cell spacing obtained by the LES is smaller than the experimental spacing, and an increase of the screech frequency is consequently expected to be observed. Nevertheless, it should be noted that the screech Strouhal number predicted by the computation remains within $10 \%$ of the values measured on rectangular jets.

The sound pressure level (SPL) of the dominant screech tone is presented in Fig. 14 as a function of the fully expanded jet Mach number $\mathbf{M}_{j}$, for the present computation and for the experiments of Panda et al. ${ }^{27}$ on rectangular jets.

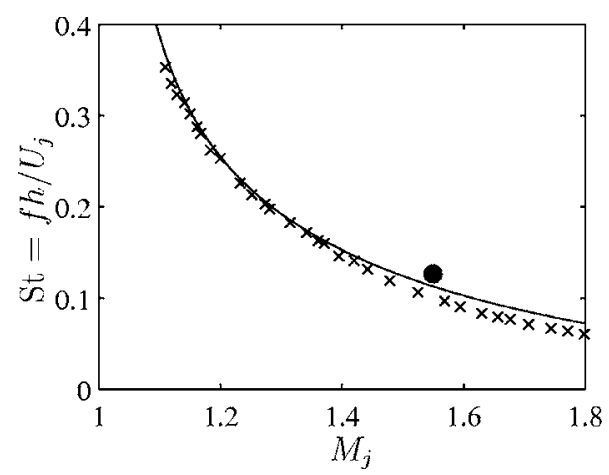

FIG. 13. Strouhal number $\mathrm{St}=f h / U_{j}$ of the fundamental screech tone as a function of the fully expanded jet Mach number $\mathrm{M}_{j}$. Measurements on rectangular jets: $\times$, Panda et al. (Ref. 27); planar jet $\left(u_{c}=0.5 U_{j}\right)$. Present computation: 


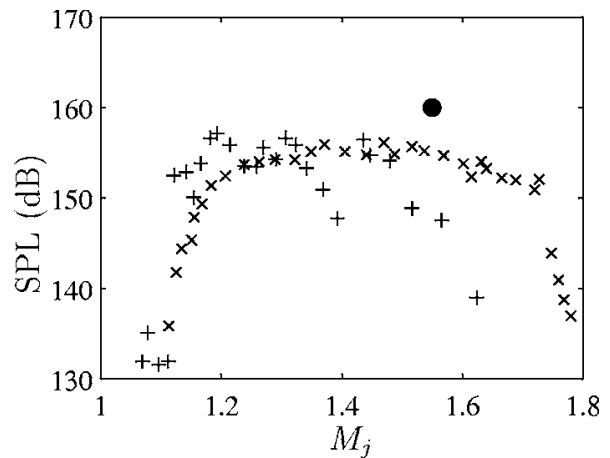

FIG. 14. Sound pressure level slightly upstream of the nozzle exit of the screech fundamental tone as a function of the fully expanded jet Mach number $\mathrm{M}_{j}$. $\times$, Panda et al. (Ref. 27) experiments on rectangular jets. $\bullet$, present computation at $P^{+}$(see Fig. 6 for locations of measurement points).

Location of the SPL measurements made by Panda $e t a l^{27}$ is not precisely defined, but the authors nevertheless indicate that their microphone was placed slightly upstream of the nozzle exit. For the present jet, the SPL is evaluated at the point $P^{+}$in Fig. 6, which will be considered as being located slightly upstream of the nozzle exit. A difference of $5 \mathrm{~dB}$ is visible between the SPL provided by the LES and the SPL obtained in the experiments of Panda et $a l^{27}$ This discrepancy may be attributed to the shock strengths. Experimental results indeed suggest that the dominant screech sources are located for rectangular jets between the third and fourth shock-cells. ${ }^{30}$ However, as shown in Sec. III B, the planar geometry of the computation leads to shock strengths higher than those observed for rectangular jets, especially in the downstream direction. Although a connection between shock strength and screech amplitude has not been clearly settled so far, ${ }^{30}$ this observation could explain the differences in Fig. 14.

Phase difference on either side of the jet is now investigated in the upstream direction, close to the nozzle exit. The time histories of the pressure signals measured at the locations $(x / h, y / h)=(-0.4,0.85)$ and $(x / h, y / h)=(-0.4,-0.85)$, represented by the points $P^{+}$and $P^{-}$in Fig. 6, are depicted in Fig. 15 for ten periods of the screech. The fluctuations at the two locations clearly seem to be correlated and in phase opposition. This trend is strongly supported by the computation of the cross-spectrum $P_{p p}$ between the two pressure probes $P^{+}$and $P^{-}$. The corresponding amplitude $\left|P_{p p}\right|$ and phase $\varphi_{p p}$ are respectively plotted in Figs. 16(a) and 16(b) as functions

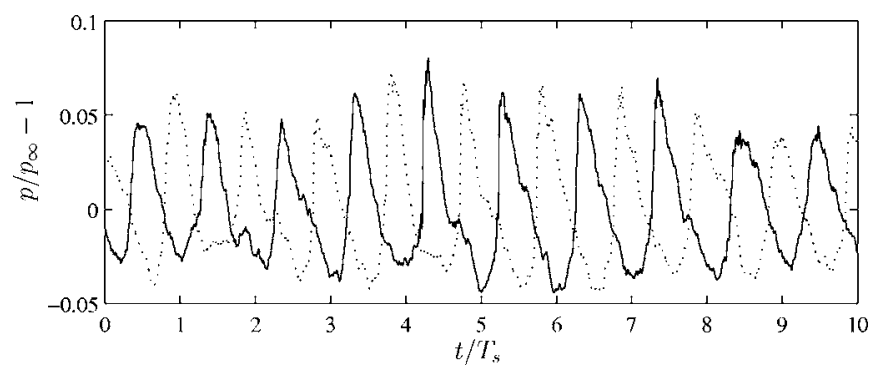

FIG. 15. Pressure history on either side of the jet in the upstream direction: - at $(x / h, y / h)=(-0.4,0.85) ; \cdots \cdots \cdots \cdot$, at $(x / h, y / h)=(-0.4,-0.85)$.
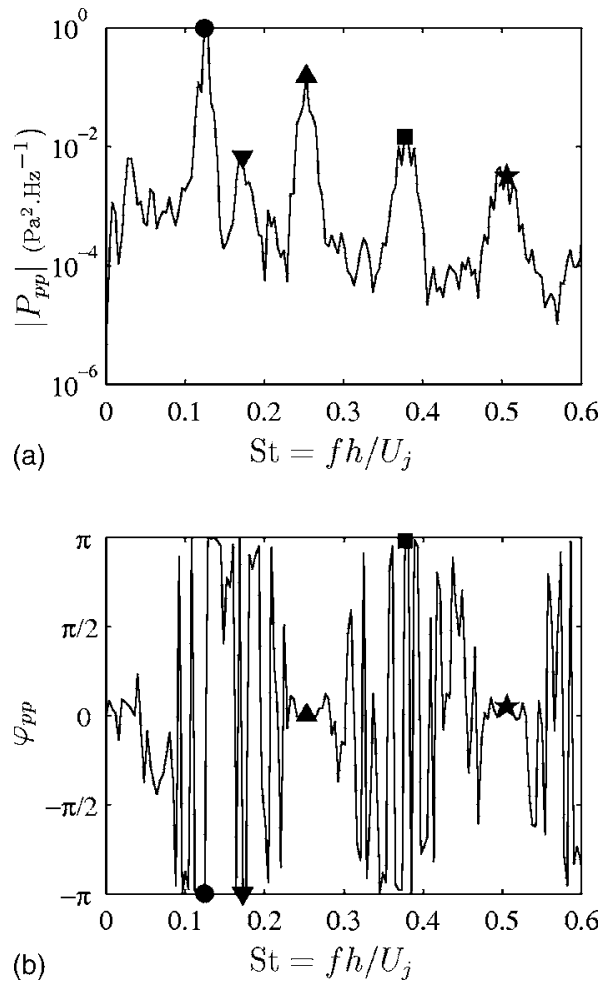

FIG. 16. Cross-spectrum of the pressure fluctuations in the upstream direction, on either side of the jet, in $(x / h, y / h)=(-0.4,0.85)$ and $(x / h, y / h)$ $=(-0.4,-0.85)$, as functions of the Strouhal number $\mathrm{St}=f h / U_{j}$. $\bullet$, Screech fundamental frequency $\left(f_{s}\right) ; \boldsymbol{\Lambda}$, first harmonic $\left(2 f_{s}\right) ; \boldsymbol{\square}$, second harmonic $\left(3 f_{s}\right) ; \star$, third harmonic $\left(4 f_{s}\right) ; \boldsymbol{\nabla}$, nonharmonic peak at $\mathrm{St}=0.171$. (a) Amplitude $\left|P_{p p}\right|$; (b) phase $\varphi_{p p}$.

of the Strouhal number $\mathrm{St}=f h / U_{j}$, and values of the coherence function $C_{p p}$ at the screech harmonic frequencies are presented in Table I. Four harmonic peaks, corresponding to the screech tones, are visible on the cross-spectrum amplitude $\left|P_{p p}\right|$. Moreover, the coherence function $C_{p p}$, which equals nearly 1 for these frequencies, ensures that the pressure signals at the two observation points are correlated. The phase difference finally indicates that the fundamental $\left(f_{s}\right)$ and the second-harmonic $\left(3 f_{s}\right)$ screech tones are antisymmetric, whereas the first $\left(2 f_{s}\right)$ and the third $\left(4 f_{s}\right)$ harmonics are symmetric. The quantity $\varphi_{p p}$ is indeed equal to $-\pi$ and $\pi$ at the frequencies $f_{s}$ and $3 f_{s}$, respectively, and is null for $f$ $=2 f_{s}$ and $f=4 f_{s}$. These results are in agreement with the measurements of Raman and Rice, ${ }^{26}$ who observed that the upstream acoustic field generated by a rectangular jet is antisymmetric for the fundamental screech tone, symmetric for the first harmonic and antisymmetric for the second harmonic.

As for the nonharmonic tone at Strouhal number St $=0.171$, a possible explanation for the emergence of this tone could rely on the hypothesis that a weak varicose mode exists inside the jet due to a bias during the computation. Nevertheless, the investigation of the cross-spectrum of the pressure signals on either side of the jet contradicts this assumption. Indeed, cross-spectrum amplitude and phase in Figs. 16(a) and 16(b) demonstrate that this tone undergoes an antisymmetric oscillation mode. At $\mathrm{St}=0.171$, the amplitude is indeed large and an investigation of the coherence function 
has shown that coherency was closed to 0.9 for this Strouhal number. The phase shift $\varphi_{p p}$ equals $\pi$, so that the pressure signals at $\mathrm{St}=0.171$ are in phase opposition.

\section{Far-field extrapolation}

A hybrid zonal approach is implemented to predict the far field noise. In this way, the near-field data provided by the LES computation are extrapolated using a simplified set of equations, allowing long-range propagation of acoustic waves.

In the present work, the three-dimensional equations of linear acoustics are solved beyond an extrapolation surface, where the LES near-field is specified. Assuming that timedependent perturbations are small and that the surrounding medium is at rest, keeping only the first-order terms in the Euler equations yields the linear acoustic equations:

$$
\frac{\partial \mathbf{U}^{\prime}}{\partial t}+\frac{\partial \mathbf{E}^{\prime}}{\partial x}+\frac{\partial \mathbf{F}^{\prime}}{\partial y}+\frac{\partial \mathbf{G}^{\prime}}{\partial z}=0,
$$

where

$$
\begin{aligned}
\mathbf{U}^{\prime} & =\left[\rho^{\prime}, \rho_{\infty} u^{\prime}, \rho_{\infty} v^{\prime}, \rho_{\infty} w^{\prime}\right] \\
\mathbf{E}^{\prime} & =\left[\rho_{\infty} u^{\prime}, p^{\prime}, 0,0\right] \\
\mathbf{F}^{\prime} & =\left[\rho_{\infty} v^{\prime}, 0, p^{\prime}, 0\right] \\
\mathbf{G}^{\prime} & =\left[\rho_{\infty} w^{\prime}, 0,0, p^{\prime}\right]
\end{aligned}
$$

The mean flow variables are $\rho_{\infty}$ and $p_{\infty}$ for the density and the pressure, respectively. Pressure fluctuations can then be computed thanks to the relationship $p^{\prime}=c_{\infty}^{2} \rho^{\prime}$, where $c_{\infty}$ $=\sqrt{\gamma p_{\infty} / \rho_{\infty}}$ is the mean sound speed, and the specific heat ratio is $\gamma=1.4$.

The numerical methods used to solve Eq. (5) are identical to those of the LES. Low-dispersion and low-dissipation explicit numerical algorithms ${ }^{15,16}$ and nonreflecting conditions $^{21}$ are thus implemented for space and time discretizations. The domain is periodic in the spanwise direction. Using undersampling and tenth-order Lagrangian interpolation the time step is doubled compared to the LES and the mesh size is multiplied by six. The computational domain contains the volume $[-35 h, 45 h] \times[0,45 h] \times[-2.5 h, 2.5 h]$.

The flow variables issued from the LES are introduced into the far-field computation at two interface planes. On these surfaces, density and velocity variables are updated with the values provided by the LES, and quantities at grid nodes close to the interfaces are computed using seven-point noncentered finite differences and filters optimized in the Fourier space. ${ }^{22}$ The first interface plane lies in the directions $x$ and $z$, and is situated on the top of the domain at $y / h=7$, while the second is in the $(y, z)$ plane at $x / h=-0.4$ and is used to take into account the acoustic field radiated in the upstream direction. Furthermore, to reduce diffraction effects due to the end of the vertical plane close to the nozzle, a symmetry condition is enforced at the bottom of the far-field grid. It should be noted, however, that the data provided by the far-field extrapolation are not relevant for low angles of observation, less than $30^{\circ}$ with respect to the jet direction.

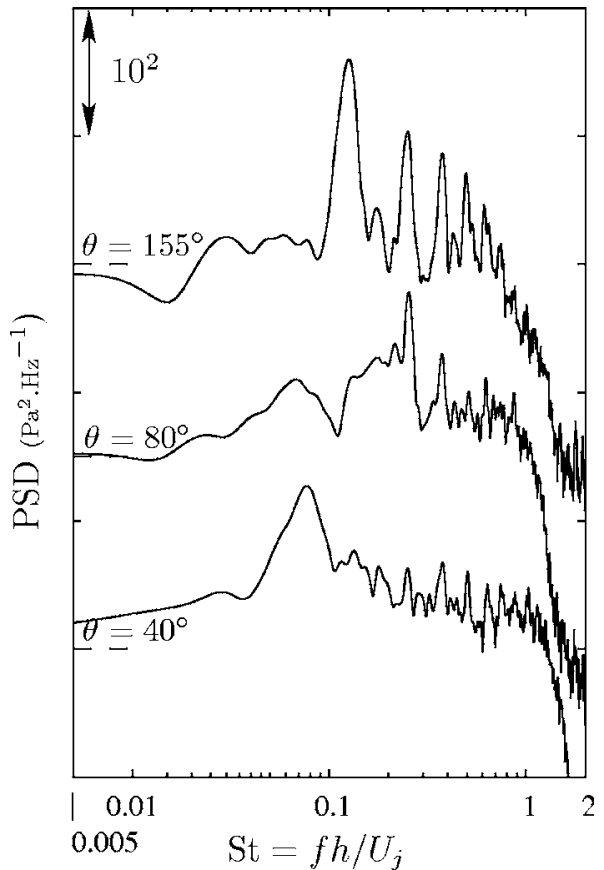

FIG. 17. Power spectral density of the pressure fluctuations in the acoustic far field as a function of the Strouhal number $\mathrm{St}=f h / U_{j}$, for various angles of observation $\theta$ with respect to the downstream direction. From the top: $\theta=155^{\circ}, \theta=80^{\circ}$, and $\theta=40^{\circ}$.

The interface $(x, z)$ plane indeed extends over $0<x / h<25$, whereas the far field is computed up to $x / h=45$.

The power spectral densities of the pressure fluctuations are computed for various positions in the far field. Measurement points are located in the central $(x, y)$ plane, on a circle of radius $35 h$ centered on the position $(x / h, y / h)=(6,0)$, which is close to the third shock-cell. The angle of observation with respect to the downstream direction is denoted $\theta$. Power spectral densities are plotted in Fig. 17 as functions of the Strouhal number $\mathrm{St}=f h / U_{j}$, for $\theta=155^{\circ}, \theta=80^{\circ}$, and $\theta$ $=40^{\circ}$. In the upstream direction, for $\theta=155^{\circ}$, the pressure spectrum is dominated by the screech harmonics and is similar to the spectrum of Fig. 13(a), computed in the vicinity of the nozzle. In the sideline direction, for $\theta=80^{\circ}$, the screech fundamental tone is no longer visible, whereas the first harmonic has the largest amplitude. Furthermore, two broadband peaks are noticeable. One occurs at Strouhal number $\mathrm{St} \simeq 0.07$, and may be associated with the mixing noise generated by the large turbulent structures. Another peak is located between $\mathrm{St}=0.1$ and $\mathrm{St}=0.2$ and is attributed to shockassociated noise. Further downstream, for $\theta=40^{\circ}$, turbulent mixing noise is the principal component of the acoustic field. A dominant peak centered on Strouhal number $\mathrm{St} \simeq 0.07$ is indeed observed in the spectrum. Three sources of noise therefore contribute to the acoustic far field of the present jet: screech tones, shock-associated noise, and mixing noise. These components of supersonic jet noise have been identified in the far field in several experimental works ${ }^{33-35}$ and may be characterized in particular by their radiation directivity. ${ }^{36}$ The fundamental screech tone is indeed observed to dominate in the forward arc, whereas the first harmonic contributes the most in the sideline direction. In addi- 


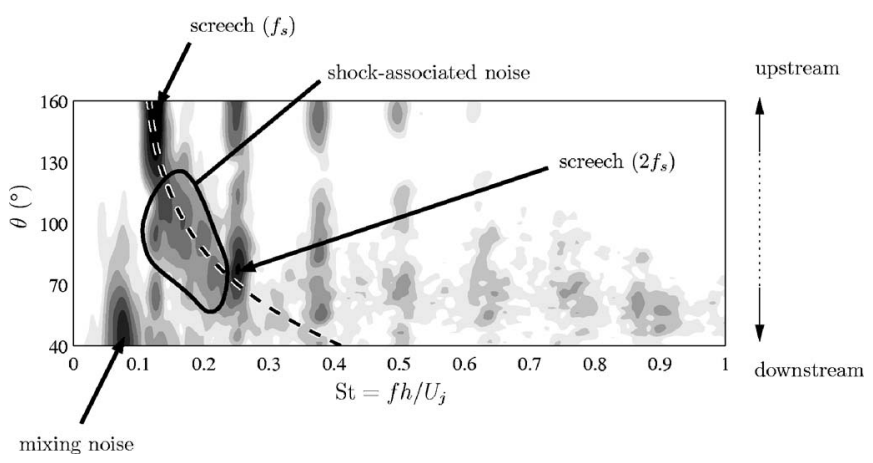

FIG. 18. Colormap of the power spectral density of the pressure perturbations in the far field as a function of the Strouhal number $\mathrm{St}=f h / U_{j}$ and of the angle of observation $\theta$ with respect to the downstream direction (logarithmic grayscale). - - -, prediction of the central frequency of shockassociated noise given by the model of Tam (Ref. 37).

tion, mixing noise is the principal noise source in the downstream direction. As for broadband shock-associated noise, it is present for a large range of angles of observation. The computed far field thus correctly reproduces the typical features of far fields radiated by supersonic jets.

This trend is further supported by Fig. 18, which represents a colormap of the power spectral density of the pressure fluctuations in the far field in logarithmic scale, as a function of the Strouhal number $\mathrm{St}=f h / U_{j}$ and of the angle of observation $\theta$ with respect to the downstream direction. Also shown in the figure is the predicted central frequency of shock-associated noise based on the model of Tam. ${ }^{37}$ The fundamental screech tone appears to radiate in the upstream direction, for instance at $\theta \simeq 150^{\circ}$ at $\mathrm{St} \simeq 0.13$, whereas the first harmonic dominates other noise sources in the sideline direction, for $\theta \simeq 80^{\circ}$. Moreover, the mixing noise generated by large scale structures emerges in the downstream direction, for $\theta<70^{\circ}$, at a Strouhal number close to 0.07 . Shockassociated noise is especially visible in the region $50^{\circ}<\theta$ $<130^{\circ}$, where a broadband peak exhibits a central frequency varying with the angle of observation. In addition, the evolution of this central frequency with $\theta$ is in agreement with the prediction of Tam. ${ }^{37}$ The acoustic far field extrapolated from the LES computation is thus in qualitative agreement with experimental data. ${ }^{36}$ Remark, furthermore, that quantitative prediction of the screech tone frequencies and of the central frequency of shock-associated noise is achieved.

The directivity patterns of the screech tones are now computed using the data provided by the far-field extrapolation. For comparison, screech directivity is also determined using a phased array of acoustic monopoles introduced by Powell. ${ }^{1}$ More elaborated models of shock-associated noise have been developed, especially in order to provide accurate predictions of the radiated pressure spectrum. ${ }^{37,38}$ However, in spite of its simplicity, the model of phased array of monopoles has been shown by Norum ${ }^{39}$ to be a good representation of screech directivity. In Powell's model, ${ }^{1}$ a series of sources, situated at the end of the shock-cells, whose relative phases are given by the convection process, radiate far field pressure fluctuations, which can be written as

$$
\begin{aligned}
p= & \exp \left[\frac{2 i \pi\left(c_{\infty} t-r_{0}\right)}{\lambda_{n}}\right] \sum_{j} \frac{S_{j}}{r_{j}} \\
& \times \exp \left[-2 i \pi j \frac{L_{s}}{\lambda_{n}}\left(\frac{1}{\mathrm{M}_{c}}-\cos \theta\right)\right],
\end{aligned}
$$

where $L_{s}$ is the shock spacing, $r_{0}$ is the distance between the observation point and the reference location $(x / h, y / h)$ $=(6,0), r_{j}$ is the separation between the observation point and the source $j, S_{j}$ is the amplitude of the source $j$, and $\lambda_{n}$, the wavelength of the $n$th screech harmonic, is given by the model of Tam: ${ }^{28}$

$$
\lambda_{n}=\frac{L_{s}}{n}\left(1+\frac{1}{\mathrm{M}_{c}}\right) .
$$

The convective Mach number is taken to be equal to $0.55 U_{j} / c_{\infty}$, and $\theta$ is the polar angle with respect to the downstream direction. The present study makes use of six sources with the following arbitrary amplitudes: $\left(S_{1}, \ldots, S_{6}\right)$ $=(0.5,0.5,1,0.5,0.25,0.25)$, the third shock-cell being the dominant source. Recall that the measurement points are located in the central $(x, y)$ plane, on a circle of radius $35 h$ centered on $(x / h, y / h)=(6,0)$.

Both numerical and analytical directivities are reported as functions of angle $\theta$ in Figs. 19(a)-19(c) for the fundamental tone, the first harmonic and the second harmonic, respectively. In all figures, the radiation patterns appear to collapse rather well for $\theta>30^{\circ}$. Indeed, lobe positions and relative amplitudes obtained by the extrapolation of the LES data and by the phased array of monopoles are seen to agree fairly well. The fundamental screech tone exhibits a dominant lobe in the upstream direction and two secondary lobes in the sideline direction. As for the first harmonic, two lobes of equal amplitudes are noticeable, one around $\theta=80^{\circ}$ and an other with a larger width in the upstream direction. Finally, the second harmonic displays three lobes, the wider lobe being oriented toward $\theta=180^{\circ}$. As expected, for small angles, the pressure field in the shadow zone does not agree with the prediction given by the expression (7). This trend is especially visible for the fundamental tone in Fig. 19(a).

\section{FLOW VISUALIZATIONS: SHOCK/VORTEX INTERACTIONS}

Shock/vortex interactions are now investigated to highlight the screech generation mechanisms. The flow motions are visualized by plotting contour maps of the density gradient modulus $|\nabla \rho|$ in the central $(x, y)$ plane. The method, commonly referred to as numerical schlieren, enables in particular to identify the location of the shocks, where strong gradients of the flow variables are observed. Such a visualization is provided in Fig. 20, which presents an overview of the flow field in the central $(x, y)$ plane. Pressure isocontours are also shown in the near field. Wavefronts propagating in the upstream direction are visible on either side of the jet and correspond to the screech. Furthermore, the third compression shock, which is located around $x / h=6.5$, seems to be the origin point of these sound waves. The flow visualization study will therefore now focus on a region surrounding the 

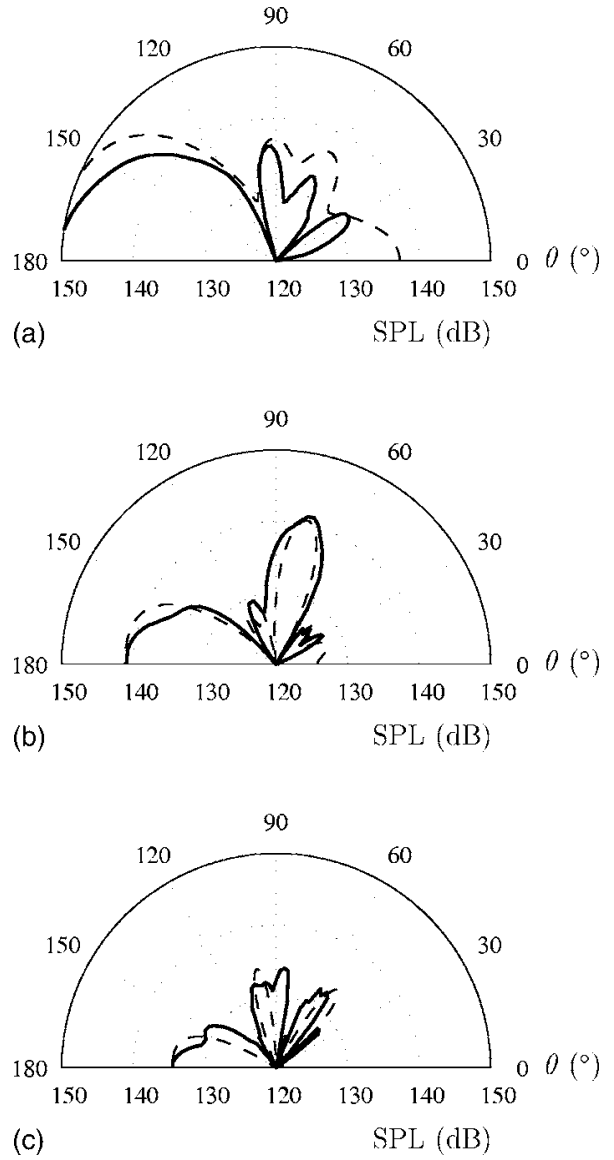

FIG. 19. Far-field directivity of screech tones. Sound pressure level (SPL) as a function of the angle of observation $\theta$ with respect to the downstream direction. - Far field extrapolation; - - , prediction by an arbitrary phased array of monopoles. (a) Screech frequency $\left(f_{s}\right)$, (b) first harmonic $\left(2 f_{s}\right)$, (c) second harmonic $\left(3 f_{s}\right)$.

third shock-cell, whose limits are given by the dashed rectangle in Fig. 20. Visualizations of shock/vortex interactions are given in Fig. 21, where half a period of the screech cycle is depicted from Figs. 21(a) to 21(e). The figures also include the upper part of the acoustic near field.

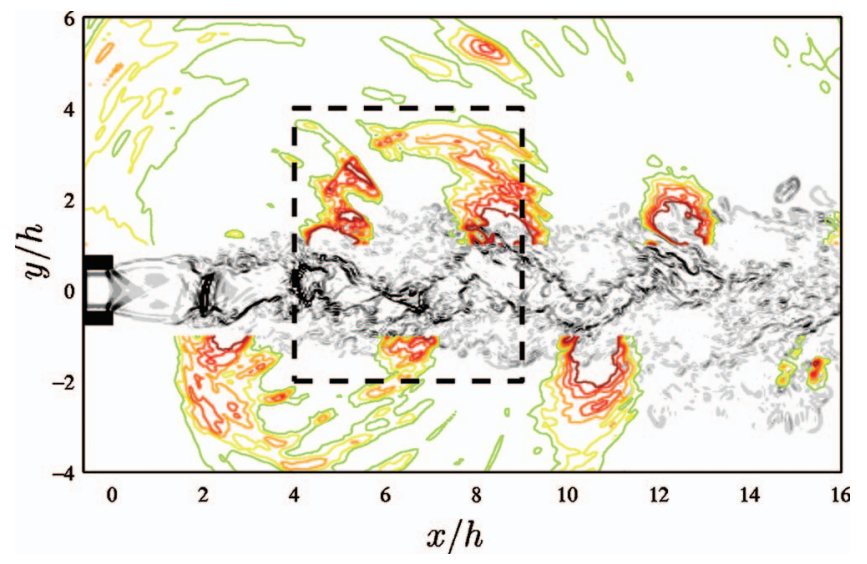

FIG. 20. (Color) Overview of the flow field and of the acoustic near field in the central $(x, y)$ plane. The density gradient modulus $|\nabla \rho|$ is plotted in gray (grayscale between 0 and $6 \rho_{\infty} / h$ ), and pressure isocontours are represented in the near field (colorscale from $p_{\infty}$ to $1.1 p_{\infty}$ ).

\section{A. Coherent structures}

As stated above, the numerical schlieren enables to locate shock positions, but strong density gradients also takes place in regions of the flow that are significantly sheared as in the jet frontier. In Fig. 21(a) for instance, dark gray regions are visible in the streamwise direction and highlight the sinuous shape of the jet induced by the self-sustained flapping mode. The coherent vortical structures associated with the screech tones excitation are also observed in the figure. The blue arrows represent the rotation and the convection of a structure located in the lower part of the jet, at $(x / h, y / h) \simeq(6.5,-1)$, and of two similar structures in the upper shear layer, at $(x / h, y / h) \simeq(4,1)$ and at $(x / h, y / h)$ $\simeq(9,1)$. These vortices are organized in an antisymmetric manner, and low-vorticity regions are found between them, as is shown, for instance, at $(x / h, y / h) \simeq(6.5,1)$ by the blue cross. These saddle points are of importance because shockleakage is expected to occur in their vicinity. ${ }^{3}$ A comparative study of the figures also shows the convection process: for instance, after half a period of the screech, the vortex in Fig. 21 (a) initially located at $(x / h, y / h) \simeq(6.5,-1)$ has moved to the position $(x / h, y / h) \simeq(9,-1)$ in Fig. $21(\mathrm{e})$. One may remark that in Fig. 21(e) flow organization is antisymmetric compared to Fig. 21(a). The blue arrows of Fig. 21(e) represent, in particular, structures antisymmetric to those visible in Fig. 21(a).

\section{B. Shock motion}

Flow visualizations in Figs. 21(a)-21(e) give, in addition, the opportunity to investigate shock motions of the third shock-cell, which is represented by a light blue dashed line. In all the figures, the shock remains located between $x / h$ $=6$ and $x / h=7$, and shows a rotating motion. In Fig. 21(a), the shock is oblique and situated around $x / h=6.5$. In Fig. 21(b), the shock is still close to $x / h=6.5$, but the upper tip of the shock has moved upstream and the lower tip downstream. This rotating motion is represented by the two light blue arrows in Fig. 21(b). Figures 21(c)-21(e) show that this motion carries on throughout the cycle, and leads to a shockcell pattern in Fig. 21(e) antisymmetric to that of Fig. 21(a): the shock in Fig. 21(e) is located at $x / h=6.5$ and exhibits an oblique shape, but with a slope opposite to that in Fig. 21(a).

\section{Sound generation}

The sound generation mechanisms are now investigated in term of the shock-leakage phenomenon. The emphasis is therefore put on the interactions between the compression shock and the low-vorticity region initially located at $(x / h, y / h) \simeq(6.5,1)$ in Fig. 21 (a). The flow motion implies that the low-vorticity region is convected downstream while the upper tip of the shock moves upstream. As a result, the low-vorticity region and the shock tip are seen to interact in Fig. 21(b), and the coupling results in the production of an acoustic wavefront, which is represented by a dashed blue line. The shock-leakage mechanism proposed by Suzuki and Lele $^{3}$ states that the shock leaks through the shear layer near the saddle points of vorticity, and creates a pressure gradient 

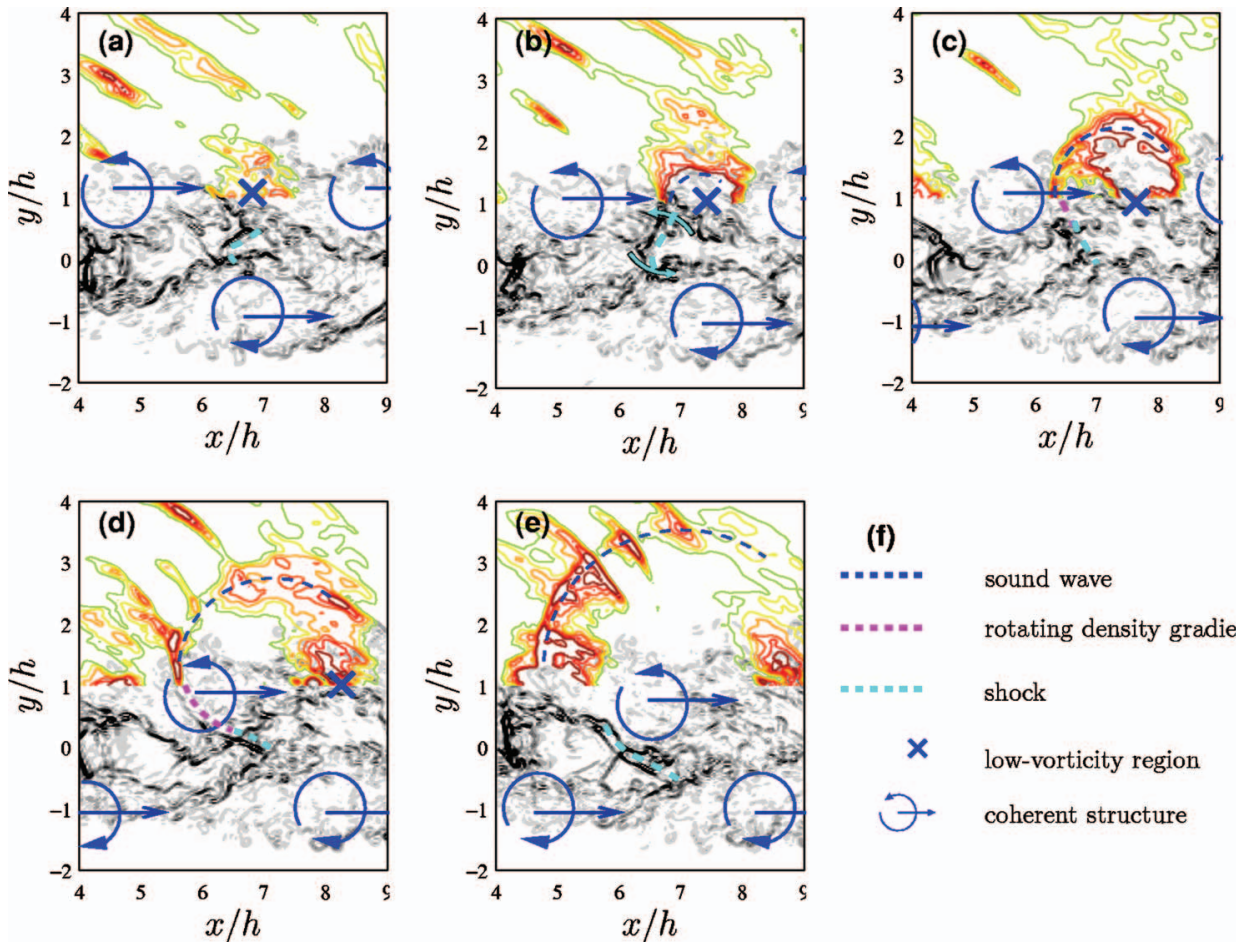

sound wave

rotating density gradient

shock

low-vorticity region

coherent structure
FIG. 21. (Color) Visualizations of the flow field and of the acoustic near field in the central $(x, y)$ plane, during half a period of the screech loop. The density gradient modulus $|\nabla \rho|$ is plotted in gray (grayscale between 0 and $6 \rho_{\infty} / h$ ), and pressure isocontours are represented in the near field (colorscale from $p_{\infty}$ to $1.1 p_{\infty}$ ). From (a) to (e): $t / T_{s}=0,1 / 8,2 / 8,3 / 8$, and $4 / 8$, where $T_{s}$ is the screech period. (f) Figure legend. that propagates in the near field with a nearly circular wavefront, covering a wide range of angles including the upstream direction. This sound generation mechanism is observed in Figs. 21(c)-21(e), where the wavefront generated in Fig. 21(c) forms a circular sound wave propagating in the upstream direction. The maximum amplitude, moreover, occurs in this direction.

Another aspect of the geometrical interpretation of $\mathrm{Su}-$ zuki and Lele ${ }^{3}$ of the shock/vortex interaction is the rotating effect of local vorticity on the wavefront. This phenomenon can be observed in the present flow visualizations. Indeed, it has been shown above that the compression shock leaks through the shear layer, but it seems in addition that the shock generates a wave that is trapped inside the vortical structure. In Figs. 21(c) and 21(d) a strong density gradient represented by a magenta dashed line is for instance seen in the core of a coherent structure. The wavefront moves upstream, but remains inside the vortex due to its rotating motion.

One may finally remark that a high frequency noise component is radiated in the downstream direction at an angle of approximately $40^{\circ}$ from the jet axis. This sound component is especially visible in Figs. 21(c) and 21(d) and seems to be generated upstream the second shock-cell. This radiation occurring at a Strouhal number of about 1 is probably a part of the mixing noise and could be due to a vortex pairing process occurring in the early development of the jet shear layer. Its contribution to the far-field spectrum in Fig. 17 is visible at $\theta=40^{\circ}$ but is, however, weak compared to the dominant contribution at $\mathrm{St}=0.09$.

\section{CONCLUSION}

In the present paper, the compressible large eddy simulation of a three-dimensional planar underexpanded jet is performed using explicit filtering with spectral-like resolution and low-dispersion and low-dissipation numerical methods. The investigation of the numerical results demonstrates that the computation reproduces the screech tone generation phenomenon, which shows the consistency of the present LES. In particular, shock-cell spacing, screech frequency, amplitude, and phase difference on either side of the jet compare correctly with experimental data provided by the literature, and possible discrepancies have been identified and attributed to the computation geometry or to the shear layer initial thickness. In addition, extrapolation of the LES near field data to the far field establishes that the noise radiated by the present jet exhibits typical features of supersonic jet noise, including three characteristic sources of noise, i.e., screech tones, shock-associated noise, and mixing noise, with frequencies and directivity patterns in agreement with experimental and theoretical data.

Flow visualizations of the third shock-cell motions finally provide evidences that shock-leakage occurs between coherent vortices generated by the screech feedback loop, resulting in the production of upstream-propagating sound waves. This sound production mechanism was first proposed by Suzuki and Lele ${ }^{3}$ to interpret acoustic radiation observed for a weak shock interacting with a shear layer. The present computation thus shows that the theory of Suzuki and Lele ${ }^{3}$ may be valid for screeching jet, and that the shock-leakage hypothesis could be used as a starting point to develop models allowing quantitative predictions of screech amplitude.

The compressible LES strategy presented in this paper hence turns out to be a reliable tool to investigate the mechanisms underlying supersonic jet noise production. Even though there remains quantitative discrepancies as in previous studies due to the limitations of the simulations, ${ }^{9-11}$ this numerical model has nonetheless been able to provide in- 
sightful details on screech noise generation. In addition, contrary to former works, the full variety of aeroacoustic phenomena occurring in an underexpanded jet such as mixing noise, shock-associated noise and screech tones, have been calculated.

\section{ACKNOWLEDGMENTS}

This work was done in the framework of the "Noise Generation in Turbulent Flows" project initiated by DFG/ CNRS. The authors gratefully acknowledge the Institut du Développement et des Ressources en Informatique Scientifique (IDRIS-CNRS) and the Commissariat à l'Énergie Atomique (CEA) for providing computing time.

${ }^{1}$ A. Powell, "On the mechanism of choked jet noise," Proc. Phys. Soc. London, Sect. B 66, 1039 (1953).

${ }^{2}$ G. Raman, "Supersonic jet screech: half-century from Powell to the present," J. Sound Vib. 225, 543 (1999).

${ }^{3}$ T. Suzuki and S. K. Lele, "Shock leakage through an unsteady vortexladen mixing layer: application to jet screech," J. Fluid Mech. 490, 139 (2003).

${ }^{4}$ H. Shen and C. K. W. Tam, "Three-dimensional numerical simulation of the jet screech phenomenon," AIAA J. 40, 33 (2002).

${ }^{5} \mathrm{X}$. Li and J. Gao, "Numerical simulation of the generation mechanism of axisymmetric supersonic jet screech tones," Phys. Fluids 17, 085105 (2005).

${ }^{6}$ C. Bogey, C. Bailly, and D. Juvé, "Noise investigation of a high subsonic, moderate Reynolds number jet using a compressible LES," Theor. Comput. Fluid Dyn. 16, 273 (2003)

${ }^{7}$ C. Bogey and C. Bailly, "Investigation of downstream and sideline subsonic jet noise using large eddy simulation," Theor. Comput. Fluid Dyn. 20, 23 (2006).

${ }^{8}$ D. J. Bodony and S. K. Lele, "On using large-eddy simulation for the prediction of noise from cold and heated turbulent jets," Phys. Fluids 17, 085103 (2005).

${ }^{9}$ I. M. A. Al-Qadi and J. N. Scott, "High-order three-dimensional numerical simulation of a supersonic rectangular jet," presented at the 9th AIAA/ CEAS Aeroacoustics Conference, Hilton Head, SC, May 12-14, 2003, AIAA Paper 2003-3238.

${ }^{10} \mathrm{~B}$. Imamoglu and P. Balakumar, "Three dimensional computation of shock induced noise in imperfectly expanded supersonic jets," presented at the 9th AIAA/CEAS Aeroacoustics Conference, Hilton Head, SC, May 12-14, 2003, AIAA Paper 2003-3249.

${ }^{11}$ C. Y. Loh, A. Himansu, and L. S. Hultgren, "A 3-D CE/SE Navier-Stokes solver with unstructured hexahedral grid for computation of near field jet screech noise," presented at the 9th AIAA/CEAS Aeroacoustics Conference, Hilton Head, SC, May 12-14, 2003, AIAA Paper 2003-3207.

${ }^{12}$ E. Garnier, M. Mossi, P. Sagault, P. Comte, and M. Deville, "On the use of shock-capturing schemes for large-eddy simulation," J. Comput. Phys. 153, 273 (1999)

${ }^{13}$ S. Ghosal, "An analysis of numerical errors in large-eddy simulations of turbulence," J. Comput. Phys. 125, 187 (1996).

${ }^{14}$ C. Bogey and C. Bailly, "Large eddy simulations of transitional round jets: influence of the Reynolds number on flow development and energy dissipation," Phys. Fluids 18, 065101 (2006).

${ }^{15} \mathrm{C}$. Bogey and C. Bailly, "A family of low-dispersive and low-dissipative explicit schemes for flow noise and noise computations," J. Comput. Phys. 194, 194 (2003).

${ }^{16}$ J. Berland, C. Bogey, and C. Bailly, "Low-dissipation and low-dispersion fourth-order Runge-Kutta algorithm," Comput. Fluids 35, 1459 (2006).

${ }^{17}$ B. Vreman, B. Geurts, and J. G. M. Kuerten, "Subgrid-modeling in LES of compressible flow," Appl. Sci. Res. 54, 191 (1995).

${ }^{18} \mathrm{C}$. Bogey and C. Bailly, "Large eddy simulations of round free jets using explicit filtering with/without dynamic Smagorinsky model," Int. J. Heat Mass Transfer 27, 603 (2006).

${ }^{19} \mathrm{C}$. Bogey and C. Bailly, "Decrease of the effective Reynolds number with eddy-viscosity subgrid-scale modeling," AIAA J. 43, 437 (2005).

${ }^{20}$ D. P. Rizzetta, M. R. Visbal, and G. A. Blaidell, "A time-implicit compact differencing and filtering scheme for large-eddy simulation," Int. J. Numer. Methods Fluids 42, 665 (2003).

${ }^{21} \mathrm{C}$. K. W. Tam and Z. Dong, "Radiation and outflow boundary conditions for direct computation of acoustic and flow disturbances in a nonuniform mean flow," J. Comput. Acoust. 4, 175 (1996).

${ }^{22}$ J. Berland, C. Bogey, O. Marsden, and C. Bailly, "High-order low dispersive and low dissipative explicit schemes for multiple-scale and boundary problems," J. Comput. Phys. 224, 637 (2007).

${ }^{23}$ T. J. Poinsot and S. K. Lele, "Boundary conditions for direct simulations of compressible viscous flows," J. Comput. Phys. 101, 104 (1992).

${ }^{24}$ A. Krothapalli, Y. Hsia, D. Baganoff, and K. Karamcheti, "The role of screech tones in mixing of an underexpanded rectangular jet," J. Sound Vib. 106, 119 (1986)

${ }^{25}$ K. B. M. Q. Zaman, "Far field noise of a subsonic jet under controlled excitation," J. Fluid Mech. 152, 83 (1985).

${ }^{26}$ G. Raman and E. J. Rice, "Instability modes excited by natural screech tones in a supersonic rectangular jet," Phys. Fluids 6, 3999 (1994).

${ }^{27}$ J. Panda, G. Raman, and K. B. M. Q. Zaman, "Underexpanded screeching jets from circular, rectangular and elliptic nozzles," presented at the 3rd AIAA/CEAS Aeroacoustics Conference, Atlanta, GA, May 12-14, 1997, AIAA Paper 1997-1623.

${ }^{28} \mathrm{C}$. K. W. Tam, "The shock-cell structures and screech tone frequencies of rectangular and non-axisymmetric supersonic jets," J. Sound Vib. 121, 135 (1998).

${ }^{29}$ P. J. Morris, T. R. S. Bhat, and G. Chen, "A linear shock-cell model for jets of arbitrary exit geometry," J. Sound Vib. 132, 199 (1989).

${ }^{30}$ G. Raman, "Cessation of screech in underexpanded jets," J. Fluid Mech. 336, 69 (1997).

${ }^{31} \mathrm{G}$. Raman, "Screech tones from rectangular jets with spanwise oblique shock-cell structures," J. Fluid Mech. 330, 141 (1997).

${ }^{32}$ F. O. Thomas and K. M. K. Prakash, "An experimental investigation of the natural transition of an untuned jet," Phys. Fluids A 3, 90 (1991).

${ }^{33}$ H. K. Tanna, "An experimental study of jet noise. Part II: shock associated noise," J. Sound Vib. 50, 429 (1977).

${ }^{34}$ T. D. Norum and J. M. Seiner, "Measurements of mean static pressure and far field acoustics of shock-containing supersonic jets," NASA Tech. Memo. TM-84521, 1 (1982).

${ }^{35}$ A. Krothapalli, P. T. Soderman, C. S. Allen, J. A. Hayes, and S. M. Jaeger, "Flight effects on the far field noise of a heated supersonic jet," AIAA J. 35, 952 (1997).

${ }^{36}$ C. K. W. Tam, "Supersonic jet noise," Annu. Rev. Fluid Mech. 27, 17 (1995).

${ }^{37}$ C. K. W. Tam, "Stochastic model theory of broadband shock associated noise from supersonic jets," J. Sound Vib. 116, 265 (1987).

${ }^{38}$ S. K. Lele, "Phased array models of shock-cell noise sources," presented at the 11th AIAA/CEAS Aeroacoustics Conference, Monterey, CA, May 23-25, 2005, AIAA Paper 2005-2841

${ }^{39}$ T. D. Norum, "Screech suppression in supersonic jets," AIAA J. 21, 235 (1983). 